\title{
Importance sampling for non-Markovian tandem queues using subsolutions
}

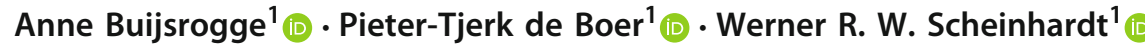

Received: 8 November 2018 / Revised: 15 June 2019 / Published online: 16 July 2019

(c) The Author(s) 2019

\begin{abstract}
In this paper, we use importance sampling simulation to estimate the probability that the number of customers in a $d$-node $G I|G I| 1$ tandem queue reaches some high level $N$ in a busy cycle of the system. We present a state-dependent change of measure for a $d$-node $G I|G I| 1$ tandem queue based on the subsolution approach, and we prove, under a mild conjecture, that this state-dependent change of measure gives an asymptotically efficient estimator for the probability of interest when all supports are bounded.
\end{abstract}

Keywords $G I|G I| 1$ queues · Tandem queues · Rare event simulation · Importance sampling

Mathematics Subject Classification 60K25 · 65C05

\section{Introduction}

In this paper, we will develop a simulation scheme to estimate the probability that the total number of customers in a $d$-node $G I|G I| 1$ tandem queue exceeds some high level $N$ in a busy cycle of the system. Two of the main methods used for rare event simulation are importance sampling and splitting: in this paper, we consider importance sampling. In importance sampling, we make the event of interest less rare by changing the underlying probability distribution.

\footnotetext{
Anne Buijsrogge

a.buijsrogge@utwente.nl

Pieter-Tjerk de Boer

p.t.deboer@utwente.nl

Werner R. W. Scheinhardt

w.r.w.scheinhardt@utwente.nl

1 University of Twente, Enschede, The Netherlands
} 
Importance sampling for queueing networks has been studied since 1989, when a state-independent change of measure for general queueing networks was proposed by Parekh and Walrand [11]. Sadowsky [12] showed that for the single queue this change of measure gives a so-called asymptotically efficient estimator. Afterwards, in [10], it has been shown that the change of measure that was proposed by Parekh and Walrand does not necessarily give an asymptotically efficient estimator for the two-node Markovian tandem queue. In [3,10], necessary and sufficient conditions for asymptotic efficiency have been provided; thus, an extension to a state-dependent change of measure is required in order to get an asymptotically efficient estimator for all input parameters.

The subsolution approach is a method, developed by Dupuis and Wang [7], which can be used to construct a state-dependent change of measure. For the two-node Markovian tandem queue [6] and Jackson networks [8], this method has been applied to develop a state-dependent change of measure that gives an asymptotically efficient estimator for the probability of interest. Note that the subsolution approach is not limited in its use to importance sampling. It can also be used for splitting, see, for example, [5], but for this paper we choose not to address splitting and focus on importance sampling instead.

In [2], it has been shown that also for non-Markovian tandem queues an extension to a state-dependent change of measure is required in order to get an asymptotically efficient estimator. In that paper, necessary conditions for asymptotic efficiency have been provided. In this paper, we extend the existing work on $d$-node Markovian tandem queues to $d$-node non-Markovian tandem queues. This extension is important, because in many practical applications the Markov assumption is not realistic.

To construct a state-dependent change of measure for non-Markovian tandem queues using the subsolution approach, we extend the state description for Markovian tandem queues - consisting of the number of customers in each queue-with the residual inter-arrival and service times of all queues. By doing so, we have complete knowledge on the state of the system and it turns out that this is sufficient information to construct a state-dependent change of measure.

Firstly, we will analyse how the subsolution approach works for the single $G I|G I| 1$ queue. Using this approach, we find the same change of measure as in [12], with a much shorter proof of asymptotic efficiency than the proof in [12]. Secondly, we consider the two-node $G I|G I| 1$ tandem queue. In that case, the state description is relatively small and therefore the proofs are still quite clean. We end with statements for the $d$-node $G I|G I| 1$ tandem queue, where we present the results but omit the proofs (which are natural extensions of the proofs for the two-node tandem queue).

The contribution of this paper is threefold. Firstly, we extend the subsolution approach from Markovian tandem queues to non-Markovian tandem queues and we present a state-(in)dependent change of measure that may give an asymptotically efficient estimator. Secondly, we prove asymptotic efficiency of the estimator for the single queue when the change of measure is based on this subsolution. For the $d$-node tandem queue, we prove that — when all supports are bounded and some conjecture holds — this state-dependent change of measure indeed gives an asymptotically efficient estimator. Lastly, we provide numerical examples to support our results. 
This paper is structured as follows: In Sect. 2, we introduce the model and notation and we provide some background knowledge about the subsolution approach and importance sampling. Then, in Sect. 3 we present a state-dependent change of measure and we prove that this change of measure gives an asymptotically efficient estimator for the probability of interest. Section 4 concludes the paper with some numerical results.

\section{Model and preliminaries}

\subsection{The model}

In this paper, we consider a $d$-node $G I|G I| 1$ tandem queue; see Fig. 1 for a graphical illustration. The inter-arrival times are i.i.d. and distributed according to some distribution $A$ and the service times of queue $j$ are i.i.d. and distributed according to some distribution $B^{(j)}$. All processes are assumed to be independent, and services at all queues follow a first-come first-served (FCFS) discipline. For notational convenience, we will denote the cumulative distribution function of any random variable $X$ by $F_{X}(x)$, its moment generating function by $M_{X}(t)$ and the log-moment generating function by $\Lambda_{X}(t)$.

We are interested in the probability of the event that the total number of customers in the system reaches some high level $N$ in a busy cycle of the system.

Throughout this paper, we make the following assumptions with respect to the distributions of the inter-arrival time and service times.

Assumption 1 (1) The moment generating functions for all service time distributions exist, i.e., for all $j, M_{B^{(j)}}(t)>0$ for some $t>0$;

(2) The system is stable, i.e., for all $j, \mathbb{E}\left[B^{(j)}\right]<\mathbb{E}[A]$;

(3) The probability of interest is non-trivial, i.e., for at least one queие $j$ we have $\mathbb{P}\left(B^{(j)}>A\right)>0$ and $\mathbb{P}\left(A>\sum_{j=1}^{d} B^{(j)}\right)>0$.

In addition, when considering the $d$-node tandem queue for $d>1$, we make the following assumption.

Assumption 2 The supports of all distributions are bounded, i.e., there exist constants $Q^{(j)}<\infty \forall j=0, \ldots$, d such that $\mathbb{P}\left(A_{k}<Q^{(0)}\right)=1, \mathbb{P}\left(B_{k}^{(j)}<Q^{(j)}\right)=1$.

When a Markovian system was studied in [6], the state description consisted of the number of customers in each queue. For a non-Markovian queue, we extend this state description by adding the residual inter-arrival time and the residual service times. Therefore, for the $d$-node tandem queue the state description is a vector with $2 d+1$

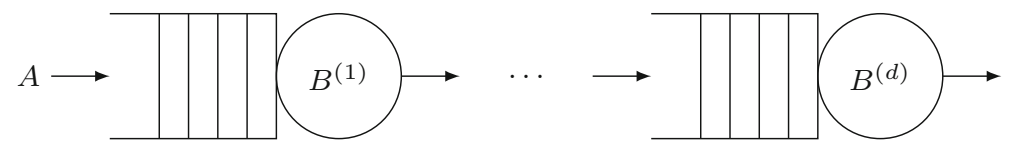

Fig. 1 The $d$-node tandem queue 
components. As in [6], we define all processes embedded at a transition for the number of customers in a queue. For any vector $\mathbf{y}$ with $2 d+1$ components $y_{1}, \ldots, y_{2 d+1}$, we introduce shorthand notation $\bar{y}_{j}=y_{d+1+j}, j=0, \ldots, d$. Using this, we let

$$
\mathbf{Z}_{i}=\left(Z_{1, i}, \ldots, Z_{d, i}, \bar{Z}_{0, i}, \ldots, \bar{Z}_{d, i}\right)
$$

denote the state of the system after $i$ transitions. Here, $Z_{j, i}, j=1, \ldots, d$, is the number of customers in queue $j$ after $i$ transitions, $\bar{Z}_{0, i}$ is the residual inter-arrival time after $i$ transitions and $\bar{Z}_{j, i}, j=1, \ldots, d$, is the residual service time at queue $j$ after $i$ transitions. If $Z_{j, i}=0$ for some $j$, then we set $\bar{Z}_{j, i}=0$. Throughout this paper, we let $\mathbf{Z}_{0}=(1,0, \ldots, 0)$, i.e., we start with one customer in queue 1 . We have $\mathbf{Z}_{i+1}=\mathbf{Z}_{i}+V_{Z}\left(\mathbf{Z}_{i}\right)$, where $V_{Z}\left(\mathbf{Z}_{i}\right)$ denotes the next transition when the state of the system after the $i$ th transition is $\mathbf{Z}_{i}$. For $i>0$, we define $V_{Z}\left(\mathbf{Z}_{i}\right)$ in terms of the shortest residual time $\mathcal{Z}\left(\mathbf{Z}_{i}\right)=\min _{k \in\{0\} \cup\left\{j: Z_{j, i}>0\right\}}\left\{\bar{Z}_{k, i}\right\}$, i.e., the minimum of the residual inter-arrival time and the residual service times of all customers in service. In other words, $\mathcal{Z}\left(\mathbf{Z}_{i}\right)$ is the time until the next transition. In Remark 1 , we make a convention about what happens when $\mathcal{Z}\left(\mathbf{Z}_{i}\right)$ is not uniquely defined.

The possible transitions when the current state is $\mathbf{Z}_{i}$, corresponding to the cases in (1), are an arrival at queue 1 , a customer going from queue $j$ to queue $j+1$, $j \in\{1, \ldots, d-1\}$ and a departure from queue $d$, which is a departure from the system. As the process starts at $(1,0, \ldots, 0)$, we need a different transition for $\mathbf{Z}_{0}$. It will become clear in Remarks 6 and 7 why this technicality is needed.

Let $\mathbf{e}_{j}$ denote the $j$ th unit vector. Then, for all $i>0$ we have

$$
\begin{aligned}
& V_{Z}\left(\mathbf{Z}_{i}\right)=-\mathcal{Z}\left(\mathbf{Z}_{i}\right)\left(\overline{\mathbf{e}}_{0}+\sum_{k=1}^{d} \overline{\mathbf{e}}_{k} \mathbb{1}\left\{Z_{k, i}>0\right\}\right) \\
& \quad+\left\{\begin{array}{cl}
\mathbf{e}_{1}+A_{i} \overline{\mathbf{e}}_{0}+B_{i}^{(1)} \overline{\mathbf{e}}_{1} \mathbb{1}\left\{Z_{1, i}=0\right\} & \text { if } \mathcal{Z}\left(\mathbf{Z}_{i}\right)=\bar{Z}_{0, i}, \\
\mathbf{e}_{j+1}-\mathbf{e}_{j}+B_{i}^{(j)} \overline{\mathbf{e}}_{j} \mathbb{1}\left\{Z_{j, i}>1\right\} & \text { if } \mathcal{Z}\left(\mathbf{Z}_{i}\right)=\bar{Z}_{j, i}, j=1, \ldots, d-1, \\
+B_{i}^{(j+1)} \overline{\mathbf{e}}_{j+1} \mathbb{1}\left\{Z_{j+1, i}=0\right\} & \text { if } \mathcal{Z}\left(\mathbf{Z}_{i}\right)=\bar{Z}_{d, i}, \\
-\mathbf{e}_{d}+B_{i}^{(d)} \overline{\mathbf{e}}_{d} \mathbb{1}\left\{Z_{d, i}>1\right\} &
\end{array}\right.
\end{aligned}
$$

and

$$
V_{Z}\left(\mathbf{Z}_{0}\right)=A_{0} \overline{\mathbf{e}}_{0}+B_{0}^{(1)} \overline{\mathbf{e}}_{1}
$$

where $A_{i}$ is the inter-arrival time if the $i$ th transition is an arrival. If the $i$ th transition is not an arrival, then $A_{i}=0$. Similarly, we have that $B_{i}^{(j)}$ is the service time of a customer at queue $j$ if a service is starting at queue $j$ at the $i$ th transition. If the $i$ th transition is not a service at queue $j$, then $B_{i}^{(j)}=0$. This means that, depending on the current state of the system, it is known which type of transition to take and each of them can have infinitely many possibilities in terms of the residuals (depending on the distribution).

We note that in (1) we consider $\mathbb{1}\left\{Z_{j, i}>1\right\}$ and not $\mathbb{1}\left\{Z_{j, i}>0\right\}$, since we do not need a new service time for queue $j$ when queue $j$ is empty after the transition. We 
do need a new service time for queue $j$ when a customer departs from queue $j-1$ and queue $j$ was empty before, i.e., $Z_{j, i}=0$.

Remark 1 If $\mathcal{Z}\left(\mathbf{Z}_{i}\right)$ is not uniquely defined, it is not clear in which order the transitions should happen. However, in most cases it is not important for our probability of interest which of the transitions happens first. Only if $\mathcal{Z}\left(\mathbf{Z}_{i}\right)=\bar{Z}_{0, i}=\bar{Z}_{d, i}$, i.e., an arrival happens at the same time as a departure from the system, the order does matter, and we use the convention that the departure occurs first, i.e., $\mathcal{Z}\left(\mathbf{Z}_{i}\right)=\bar{Z}_{d, i}$.

As for the Markovian system in [6,8], we will work with the scaled process. Therefore, we define

$$
\mathbf{X}_{i}=\left(X_{1, i}, \ldots, X_{d, i}, \bar{X}_{0, i}, \ldots, \bar{X}_{d, i}\right)=\frac{1}{N} \mathbf{Z}_{i}
$$

and we have

$$
\mathbf{X}_{i+1}=\mathbf{X}_{i}+\frac{1}{N} V_{X}\left(\mathbf{X}_{i}\right)
$$

where $V_{X}\left(\mathbf{X}_{i}\right)=V_{Z}\left(\mathbf{X}_{i} N\right)$ is the $(i+1)$ th transition when the state of the scaled system is $\mathbf{X}_{i}$. The advantage of the scaled system is that the first $d$ elements of the state description are always in $[0,1]$, which does not increase as $N$ increases. Note that for the scaled system we will use a similar convention as in Remark 1. Similarly to the unscaled system, we define $\mathcal{X}\left(\mathbf{X}_{i}\right)=\frac{\mathcal{Z}\left(\mathbf{X}_{i}\right)}{N}$. Hence, for $i>0$, we have that

$$
\begin{aligned}
& V_{X}\left(\mathbf{X}_{i}\right)=-\mathcal{X}\left(\mathbf{X}_{i}\right) N\left(\overline{\mathbf{e}}_{0}+\sum_{k=1}^{d} \overline{\mathbf{e}}_{k} \mathbb{1}\left\{X_{k, i}>0\right\}\right) \\
& \quad+\left\{\begin{array}{cl}
\mathbf{e}_{1}+A_{i} \overline{\mathbf{e}}_{0}+B_{i}^{(1)} \overline{\mathbf{e}}_{1} \mathbb{1}\left\{X_{1, i}=0\right\} & \text { if } \mathcal{X}\left(\mathbf{X}_{i}\right)=\bar{X}_{0, i}, \\
\mathbf{e}_{j+1}-\mathbf{e}_{j}+B_{i}^{(j)} \overline{\mathbf{e}}_{j} \mathbb{1}\left\{X_{j, i}>\frac{1}{N}\right\} & \text { if } \mathcal{X}\left(\mathbf{X}_{i}\right)=\bar{X}_{j, i}, j=1, \ldots, d-1, \\
+B_{i}^{(j+1)} \overline{\mathbf{e}}_{j+1} \mathbb{1}\left\{X_{j+1, i}=0\right\} & \text { if } \mathcal{X}\left(\mathbf{X}_{i}\right)=\bar{X}_{d, i}, \\
-\mathbf{e}_{d}+B_{i}^{(d)} \overline{\mathbf{e}}_{d} \mathbb{1}\left\{X_{d, i}>\frac{1}{N}\right\} &
\end{array}\right.
\end{aligned}
$$

and

$$
V_{X}\left(\mathbf{X}_{0}\right)=A_{0} \overline{\mathbf{e}}_{0}+B_{0}^{(1)} \overline{\mathbf{e}}_{1}
$$

where $\mathbf{X}_{0}=\left(\frac{1}{N}, 0, \ldots, 0\right)$.

Now that we have defined the full state description, we define the goal set $\delta_{e}$ and taboo set $\delta_{0}$ in the following way:

$$
\begin{aligned}
& \delta_{e}=\left\{\mathbf{x}: \sum_{k=1}^{d} x_{k}=1-\frac{1}{N}, \mathcal{X}(\mathbf{x})=\bar{x}_{0}\right\}, \\
& \delta_{0}=\left\{\mathbf{x}: x_{k}=\bar{x}_{k}=0 \forall k \in\{1, \ldots, d\}\right\},
\end{aligned}
$$


where the goal set is reached if there are $N-1$ customers in the system and the next event is an arrival to the system, and the taboo set is reached when there are no customers in the system.

Using the definitions above, we define the time to reach $\delta_{e}$ before $\delta_{0}$ as

$$
\tau_{N}=\inf \left\{i>0: \mathbf{X}_{i} \in \delta_{e}, \mathbf{X}_{k} \notin \delta_{0} \forall k=1, \ldots, i-1\right\},
$$

and we set $\tau_{N}=\infty$ when $\delta_{0}$ is reached before $\delta_{e}$. Now we can write our probability of interest, $p_{N}$, in terms of $\tau_{N}$ as

$$
p_{N}=\mathbb{P}\left(\tau_{N}<\infty\right)
$$

Remark 2 Note that we could define $\delta_{e}$ and $\delta_{0}$ differently, i.e., there are more states for which we know that the total number of customers will reach $N$ or that the system will be empty. However, the current definitions are easy to use and it turns out that these definitions are sufficient for our proofs; see Remarks 8 and 12.

From $[1,12]$, we know that the decay rate of $p_{N}$ equals

$$
\gamma=-\lim _{N \rightarrow \infty} \frac{1}{N} \log p_{N}=-\Lambda_{A}\left(-\theta^{*}\right)
$$

where $\theta^{*}=\min _{j}\left\{\theta_{j}\right\}$ with

$$
\theta_{j}=\sup \left\{\theta: \Lambda_{A}(-\theta)+\Lambda_{B^{(j)}}(\theta) \leq 0\right\}
$$

or equivalently $\theta_{j}=\sup \left\{\theta: M_{A}(-\theta) M_{B^{(j)}}(\theta) \leq 1\right\}$. Note that as a result of the stability and non-triviality assumption, $0<\theta^{*}<\infty$; see [2] for a formal proof of $\mathbb{P}\left(B^{(j)}>A\right) \Leftrightarrow \theta^{*}=\infty$. This gives rise to the following definition.

Definition 1 Queue $j$ is a bottleneck queue when $\theta_{j}=\theta^{*}$.

Which queue is the bottleneck also determines the form of the so-called most likely path to overflow: if the overflow level is reached, this is most likely done along a specific path (that dominates the probability of reaching the overflow level and hence determines the decay rate). When queue $j$ is the bottleneck queue, it is therefore expected that along the most likely path $x_{j}>0$.

\subsection{Preliminaries}

In order to estimate the probability of interest using simulation, we use importance sampling to make our event of interest less rare by changing the underlying probability distribution. In the current work, we make the change of measure state-dependent by using the subsolution approach.

\subsubsection{Subsolution approach}

The subsolution approach for importance sampling has been introduced in [7]. Later, in $[6,8]$, it has been used to find a state-dependent change of measure that results in 
an asymptotically efficient estimator for $p_{N}$ in the context of a Markovian tandem queue [6] and Jackson networks [8]. The definition of a classical subsolution is as follows.

Definition 2 A real-valued function $W(\mathbf{x})$ is called a classical subsolution if

1. $W(\mathbf{x})$ is continuously differentiable,

2. $\mathbb{H}(\mathbf{x}, \mathrm{DW}(\mathbf{x})) \geq 0$ for every $\mathbf{x}$,

3. $W(\mathbf{x}) \leq 0$ for $\mathbf{x} \in \delta_{e}$,

where

$$
\mathbb{H}(\mathbf{x}, \mathrm{DW}(\mathbf{x}))=-\log \left(\mathbb{E}\left[e^{-\left\langle\mathrm{DW}(\mathbf{x}), V_{X}(\mathbf{x})\right\rangle}\right]\right)
$$

In addition to this definition, in order to use the subsolution as a basis for a change of measure we require that

4. $W\left(\left(\frac{1}{N}, 0, \ldots, 0\right)\right)=\gamma$,

so that the change of measure can be useful (i.e., asymptotically efficient; see Definition 3). This means that along the most likely path to reach the overflow level, subsolution properties 2 and 3 are satisfied with equality; see also [8].

In [6], there is an additional condition in the definition of a classical subsolution for the boundaries, when at least one of the queues is empty, and in [8] there are dedicated boundary functions for $\mathbb{H}(\mathbf{x}, \mathrm{DW}(\mathbf{x}))$, which are used when one of the queues is empty. We will include the boundaries in $\mathbb{H}(\mathbf{x}, \mathrm{DW}(\mathbf{x}))$, as the boundaries are included in $V_{X}(\mathbf{x})$ by means of indicator functions.

Remark 3 The function $W(\mathbf{x})$ that we will construct in the sequel consists of (a combination of) affine functions so that for all these affine functions its derivative DW(x) is a constant. Whenever we consider an affine function, we will denote the derivative by $\alpha$.

\subsubsection{Importance sampling simulation}

In this paper, we will propose a particular change of measure and prove that it results in an asymptotically efficient estimator for $p_{N}$; see Definition 3 . In order to define the change of measure, we first introduce some notation for the probability measure. With some abuse of notation, we let $\mathrm{d} F_{V_{X}(\mathbf{x})}(\mathbf{v})$ be the probability measure for some random vector $V_{X}(\mathbf{x})$. Typically, the vector $V_{X}(\mathbf{x})$ contains one random variable, see (2), but it may result in zero or two random variables as well, for example, when a customer moves from queue $j$ to $j+1$ and queue $j+1$ is empty, there are two random variables in $V_{X}(\mathbf{x})$. For example, we can interpret $\mathrm{d} F_{V_{X}(\mathbf{x})}(\mathbf{v})$ as $\mathrm{d} F_{A}(a)$ if $\mathcal{X}(\mathbf{x})=\bar{x}_{0}$ and $x_{1}>0$.

Remark 4 The probability measure of $V_{X}(\mathbf{x})$ can be written in a more formal way, which we illustrate by considering the arrival transition in a single queue. Thus, consider a state $\mathbf{x}=\left(x_{1}, \bar{x}_{0}, \bar{x}_{1}\right)$ for which $x_{1}>0$ and $\mathcal{X}(\mathbf{x})=\bar{x}_{0}$. Then, we have

$$
F_{V_{X}(\mathbf{x})}\left(\mathbf{v} \mid \mathbf{x}: \bar{x}_{0}<\bar{x}_{1}, x_{1}>0\right)=\mathbb{1}\left\{v_{1} \geq 1\right\} \cdot F_{A}\left(\bar{v}_{0}+\bar{x}_{0} N\right) \cdot \mathbb{1}\left\{\bar{v}_{1} \geq-\bar{x}_{0} N\right\},
$$


and thus the density

$$
\frac{\partial^{3} F_{V_{X}(\mathbf{x})}\left(\mathbf{v} \mid \mathbf{x}: \bar{x}_{0}<\bar{x}_{1}, x_{1}>0\right)}{\partial v_{1} \partial \bar{v}_{0} \partial \bar{v}_{1}}=\delta\left(v_{1}-1\right) \cdot \mathrm{d} F_{A}\left(\bar{v}_{0}+\bar{x}_{0} N\right) \cdot \delta\left(\bar{v}_{1}+\bar{x}_{0} N\right)
$$

We remark that two of the three components of the transition $V_{X}(\mathbf{x})$ are deterministic, while the remaining component consists of a random sample from the inter-arrival time distribution $F_{A}$, added to the deterministic value $-\bar{x}_{0} N$. Also, in larger models, most components of the transition are deterministic (cf. (2)); therefore, we prefer the shorter but less precise notation in which only the random component(s) are written down.

Let $W(\mathbf{x})$ be a classical subsolution; see Sect. 2.2.1. Then, we define the change of measure as

$$
\mathrm{d} \bar{F}_{V_{X}(\mathbf{x})}(\mathbf{v} \mid \mathbf{x})=e^{\langle-\mathrm{DW}(\mathbf{x}), \mathbf{v}\rangle} e^{\mathbb{H}(\mathbf{x}, \mathrm{DW}(\mathbf{x}))} \mathrm{d} F_{V_{X}(\mathbf{x})}(\mathbf{v}),
$$

where $\bar{F}_{V_{X}(\mathbf{x})}(\mathbf{v} \mid \mathbf{x})$ is the distribution function under the new measure and $\mathbb{H}(\mathbf{x}, \mathrm{DW}(\mathbf{x}))$ is defined in (6). Note that the latter quantity can be interpreted as a normalization constant. In fact, (7) means that we apply an exponential tilt to a random variable (that depends on the random vector $V_{X}(\mathbf{x})$ ), for example, when $\mathcal{X}(\mathbf{x})=\bar{x}_{0}$ and $x_{1}>0$ we are tilting the inter-arrival times exponentially with some parameter.

While performing a simulation run and changing the underlying probability distributions at every step, we keep track of the likelihood ratio. The likelihood ratio for a successful path $\mathcal{P}=\left(\mathbf{X}_{i}, i=0, \ldots, \tau_{N}\right)$ is

$$
L(\mathcal{P})=\prod_{i=0}^{\tau_{N}-1} \frac{\mathrm{d} F_{V_{X}\left(\mathbf{X}_{i}\right)}\left(V_{X}\left(\mathbf{X}_{i}\right)\right)}{\mathrm{d} \bar{F}_{V_{X}\left(\mathbf{X}_{i}\right)}\left(V_{X}\left(\mathbf{X}_{i}\right) \mid \mathbf{X}_{i}\right)}=\prod_{i=0}^{\tau_{N}-1} e^{\left\langle\mathrm{DW}\left(\mathbf{X}_{i}\right), V_{X}\left(\mathbf{X}_{i}\right)\right\rangle} e^{-\mathbb{H}\left(\mathbf{X}_{i}, \mathrm{DW}\left(\mathbf{X}_{i}\right)\right)},
$$

where the second equality follows by using (7).

We now define the estimator of $p_{N}$ to be $L(\mathcal{P}) I(\mathcal{P})$, where $I(\mathcal{P})$ indicates whether we have reached level $N$ or not, i.e., $I(\mathcal{P})=\mathbb{1}\left\{\tau_{N}<\infty\right\}$. This estimator is unbiased under the new measure, denoted by $\mathbb{Q}$, since

$$
p_{N}=\mathbb{E}[I(\mathcal{P})]=\mathbb{E}^{\mathbb{Q}}[L(\mathcal{P}) I(\mathcal{P})]
$$

We use the following definition for asymptotic efficiency.

Definition 3 The estimator for $p_{N}$ is asymptotically efficient if and only if

$$
\liminf _{N \rightarrow \infty} \frac{\log \mathbb{E}^{\mathbb{Q}}\left[L(\mathcal{P})^{2} I(\mathcal{P})\right]}{\log p_{N}} \geq 2
$$

Using the decay rate of $p_{N}$, see (4), we find that Definition 3 is equivalent to the following definition. 
Corollary 1 The estimator for $p_{N}$ is asymptotically efficient if and only if

$$
\limsup _{N \rightarrow \infty} \frac{1}{N} \log \mathbb{E}^{\mathbb{Q}}\left[L(\mathcal{P})^{2} I(\mathcal{P})\right] \leq-2 \gamma .
$$

\section{Asymptotically efficient change of measure}

In this section, we present our main result. For readability, we present it for three cases separately. In Sect. 3.1, we start with $d=1$, i.e., the single queue, where the subsolution approach results in a state-independent change of measure. Although this change of measure has been proven to be asymptotically efficient in [12], we present an alternative proof using the method that will be extended to a state-dependent change of measure for the $d$-node tandem queue. Secondly, we consider $d=2$ in Sect. 3.2 in detail, as in this case the state vector consists of five dimensions only. Lastly, in Sect. 3.3, we present the result for the $d$-node tandem queue, but we omit the proofs since they are very similar to the two-node case.

The approach of the subsolution method, as developed in [7], is the same in all cases defined above and is as follows:

1. For all possible $\mathbf{x}$, we find solutions $\boldsymbol{\alpha}$ to $\mathbb{H}(\mathbf{x}, \boldsymbol{\alpha}) \geq 0$.

2. We construct a function $W(\mathbf{x})$ that both is continuously differentiable and satisfies properties 3 and 4 in and below Definition 2, as $N \rightarrow \infty$. The function $W(\mathbf{x})$ will be as indicated in Remark 3; more precisely, for each $\mathbf{x}$ we will have $\operatorname{DW}(\mathbf{x}) \approx \boldsymbol{\alpha}$, where $\boldsymbol{\alpha}$ is a solution corresponding to $\mathbf{x}$ as found in Step 1 .

3. We then use the function $W(\mathbf{x})$ to define a change of measure as in (7).

After we have proposed the change of measure, we will prove asymptotic efficiency of this change of measure.

\subsection{The single $G|| G|| 1$ queue}

For the single queue, the model, as presented in Sect. 2.1, simplifies significantly. We will highlight the most important simplifications. To start with, the state description reduces to $\mathbf{x}=\left(x_{1}, \bar{x}_{0}, \bar{x}_{1}\right)$. Furthermore, queue 1 is never empty during a busy cycle of the system and so $\mathcal{X}(\mathbf{x})=\min \left\{\bar{x}_{0}, \bar{x}_{1}\right\}$. Due to the same reason, we always have $\mathbb{1}\left\{x_{1}=0\right\}=0$ and, when there is a departure from the system, $\mathbb{1}\left\{x_{1}>\frac{1}{N}\right\}=1$ (otherwise, $\mathbf{x} \in \delta_{0}$, i.e., the system will become empty after the transition). In view of Remark 6 , we do not yet substitute $\mathbb{1}\left\{x_{1}=0\right\}=0$ in the remainder. Thus, (2) in the case $d=1$ becomes

$$
\begin{aligned}
V_{X}(\mathbf{x})= & -\mathcal{X}(\mathbf{x}) N\left(\overline{\mathbf{e}}_{0}+\overline{\mathbf{e}}_{1} \mathbb{1}\left\{x_{1}>0\right\}\right) \\
& + \begin{cases}\mathbf{e}_{1}+A_{i} \overline{\mathbf{e}}_{0}+B_{i}^{(1)} \overline{\mathbf{e}}_{1} \mathbb{1}\left\{x_{1}=0\right\} & \text { if } \mathcal{X}(\mathbf{x})=\bar{x}_{0}, \\
-\mathbf{e}_{1}+B_{i}^{(1)} \overline{\mathbf{e}}_{1} \mathbb{1}\left\{x_{1}>\frac{1}{N}\right\} & \text { if } \mathcal{X}(\mathbf{x})=\bar{x}_{1} .\end{cases}
\end{aligned}
$$


In the next sections, we will follow the subsolution approach step by step and conclude with a proof of asymptotic efficiency of the change of measure based on the developed subsolution.

\subsubsection{Solution to $\mathbb{H}(\mathrm{x}, \alpha) \geq 0$ for all $\mathrm{x}$}

We have, for all possible $\mathbf{x} \neq\left(\frac{1}{N}, 0,0\right)$, from (6) and the above, that

$$
\begin{aligned}
\mathbb{H}(\mathbf{x}, \boldsymbol{\alpha})= & -\log \left(\mathbb{E}\left[e^{-\left\langle\boldsymbol{\alpha}, V_{X}(\mathbf{x})\right\rangle}\right]\right) \\
= & -\mathcal{X}(\mathbf{x}) N\left(\bar{\alpha}_{0}+\bar{\alpha}_{1}\right) \\
& + \begin{cases}\alpha_{1}-\Lambda_{A}\left(-\bar{\alpha}_{0}\right)-\Lambda_{B^{(1)}}\left(-\bar{\alpha}_{1} \mathbb{1}\left\{x_{1}=0\right\}\right) & \text { if } \mathcal{X}(\mathbf{x})=\bar{x}_{0}, \\
-\alpha_{1}-\Lambda_{B^{(1)}}\left(-\bar{\alpha}_{1}\right) & \text { if } \mathcal{X}(\mathbf{x})=\bar{x}_{1},\end{cases}
\end{aligned}
$$

and

$$
\mathbb{H}\left(\left(\frac{1}{N}, 0,0\right), \boldsymbol{\alpha}\right)=-\Lambda_{A}\left(-\bar{\alpha}_{0}\right)-\Lambda_{B^{(1)}}\left(-\overline{\alpha_{1}}\right) .
$$

Then, using that $\mathbb{1}\left\{x_{1}=0\right\}=0$, a solution to $\mathbb{H}(\mathbf{x}, \boldsymbol{\alpha}) \geq 0$ for all $\mathbf{x}$ during a busy cycle of the system is

$$
\boldsymbol{\alpha}=\left(\alpha_{1}, \bar{\alpha}_{0}, \bar{\alpha}_{1}\right)=\left(-\gamma, \theta^{*},-\theta^{*}\right),
$$

where $\theta^{*}$ equals $\theta_{1}$ in (5).

Remark 5 Another solution to $\mathbb{H}(\mathbf{x}, \boldsymbol{\alpha}) \geq 0$ is $\boldsymbol{\alpha}=(0,0,0)$. As this is equivalent to no change of measure, we will not use this solution.

Remark 6 To justify the necessity of $\mathbf{X}_{0} \neq(0,0,0)$, we note that when $\mathbf{X}_{0}$ would be equal to $(0,0,0)$ our proposed solution does not satisfy $\mathbb{H}(\mathbf{x}, \boldsymbol{\alpha}) \geq 0$ for $\mathcal{X}(\mathbf{x})=\bar{x}_{0}$. Using $\mathbf{X}_{0}$ solves this issue. We will justify our specific choice of $\mathbf{X}_{0}=\left(\frac{1}{N}, 0,0\right)$ in Remark 7.

\subsubsection{Construction of $W(\mathrm{x})$}

To use the subsolution approach, we want to choose the function $W(\mathbf{x})$ such that $W(\mathbf{x}) \leq 0$ for $\mathbf{x} \in \delta_{e}$ and $W\left(\left(\frac{1}{N}, 0,0\right)\right)=\gamma$; see properties 3 and 4 in and below Definition 2. For the single queue, we have $\delta_{e}=\left\{\mathbf{x}: x_{1}=1-\frac{1}{N}, \mathcal{X}(\mathbf{x})=\bar{x}_{0}\right\}$; see (3). Combining these, together with $\operatorname{DW}(\mathbf{x})=\alpha=\left(-\gamma, \theta^{*},-\theta^{*}\right)$, we find

$$
W(\mathbf{x})=-\gamma x_{1}+\theta^{*}\left(\bar{x}_{0}-\bar{x}_{1}\right)+\gamma,
$$

satisfying all the requirements when $N \rightarrow \infty$ : indeed, $W(\mathbf{x})=-\gamma\left(1-\frac{1}{N}\right)+\theta^{*}\left(\bar{x}_{0}-\right.$ $\left.\bar{x}_{1}\right)+\gamma \leq \frac{\gamma}{N}$ for all $\mathbf{x} \in \delta_{e}$, and $W\left(\left(\frac{1}{N}, 0,0\right)\right)=\gamma\left(1-\frac{1}{N}\right)$, and so when $N \rightarrow \infty$ the boundary conditions for $W(\mathbf{x})$ are satisfied. In Fig. 2, the function $W(\mathbf{x})$ is displayed as a function of $x_{1}$ and $\bar{x}_{0}-\bar{x}_{1}$. 


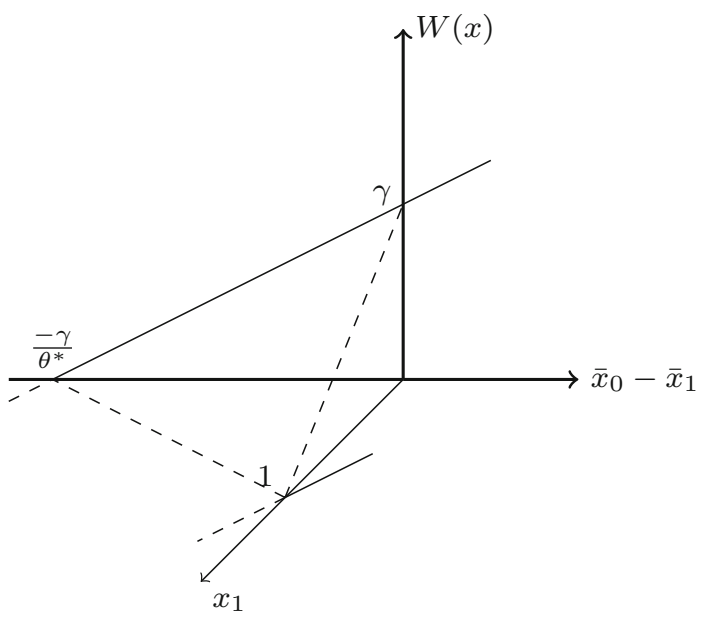

Fig. 2 The classical subsolution $W(\mathbf{x})$ for the single queue as a function of $x_{1}$ and $\bar{x}_{0}-\bar{x}_{1}$

Remark 7 To justify the specific choice of $\bar{x}_{0}=\bar{x}_{1}=0$ in $\mathbf{X}_{0}=\left(\frac{1}{N}, 0,0\right)$, we note that any other choice does not satisfy $W\left(\left(\frac{1}{N}, \bar{x}_{0}, \bar{x}_{1}\right)\right)=\gamma$ when $N \rightarrow \infty$. For the choice $x_{1}=\frac{1}{N}$, we note that this is equivalent to starting with 1 customer in the system, and hence this is a natural choice.

\subsubsection{The change of measure}

We now find that, using (7), the change of measure is, for $\mathbf{x} \neq\left(\frac{1}{N}, 0,0\right)$,

$$
\mathrm{d} \bar{F}_{V_{X}(\mathbf{x})}(\mathbf{v} \mid \mathbf{x})= \begin{cases}\frac{e^{-a \theta^{*}}}{M_{A}\left(-\theta^{*}\right)} \mathrm{d} F_{A}(a) & \text { if } \mathcal{X}(\mathbf{x})=\bar{x}_{0}, \\ \frac{e^{b_{1} \theta^{*}}}{M_{B^{(1)}}\left(\theta^{*}\right)} \mathrm{d} F_{B^{(1)}}\left(b_{1}\right) & \text { if } \mathcal{X}(\mathbf{x})=\bar{x}_{1},\end{cases}
$$

and

$$
\mathrm{d} \bar{F}_{V_{X}\left(\left(\frac{1}{N}, 0,0\right)\right)}\left(\mathbf{v} \mid\left(\frac{1}{N}, 0,0\right)\right)=\frac{e^{-a \theta^{*}+b_{1} \theta^{*}}}{M_{A}\left(-\theta^{*}\right) M_{B^{(1)}}\left(\theta^{*}\right)} \mathrm{d} F_{A}(a) \mathrm{d} F_{B^{(1)}}\left(b_{1}\right) .
$$

Proposition 1 The change of measure provided in (9) and (10) is the same change of measure as the state-independent change of measure from [12].

Proof The proof is straightforward by noting that the inter-arrival times are exponentially tilted with parameter $-\theta^{*}$ and the service times are exponentially tilted with parameter $\theta^{*}$.

\subsubsection{Asymptotic efficiency}

In [12], it has been shown that the change of measure as mentioned above is asymptotically efficient under the condition that $\mathbb{P}\left(B_{k}^{(1)}<M\right)=1$ for some finite constant $M$. 
This restriction to bounded service times is said to be a technicality, but the paper is not clear about how to remove it. Instead, we will give a different proof without the need for this condition.

Theorem 1 Under Assumption 1, the state-independent change of measure based on $D W(\mathbf{x})=\left(-\gamma, \theta^{*},-\theta^{*}\right)$, see (9) and (10), is asymptotically efficient for the single GI $|G I| 1$ qиеие.

Proof We start with the $\log$ likelihood, $\log L(\mathcal{P})$, of any path $\mathcal{P}=\left(\mathbf{X}_{i}, i=0, \ldots, \tau_{N}\right)$ such that $\tau_{N}<\infty$. By using (8), we find

$$
\begin{aligned}
\log L(\mathcal{P}) & =N \sum_{i=0}^{\tau_{N}-1}\left\langle\mathrm{DW}\left(\mathbf{X}_{i}\right), \mathbf{X}_{i+1}-\mathbf{X}_{i}\right\rangle-\sum_{i=0}^{\tau_{N}-1} \mathbb{H}\left(\mathbf{X}_{i}, \mathrm{DW}\left(\mathbf{X}_{i}\right)\right) \\
& \leq N \sum_{i=0}^{\tau_{N}-1}\left\langle\mathrm{DW}\left(\mathbf{X}_{i}\right), \mathbf{X}_{i+1}-\mathbf{X}_{i}\right\rangle \\
& =N\left\langle\mathrm{DW}\left(\mathbf{X}_{0}\right), \mathbf{X}_{\tau_{N}}-\mathbf{X}_{0}\right\rangle \\
& =N\left(-\gamma\left(1-\frac{1}{N}-\frac{1}{N}\right)+\theta^{*}\left(\bar{X}_{0, \tau_{N}}-\bar{X}_{0,0}\right)-\theta^{*}\left(\bar{X}_{1, \tau_{N}}-\bar{X}_{1,0}\right)\right) \\
& =N\left(-\gamma\left(1-\frac{2}{N}\right)+\theta^{*}\left(\bar{X}_{0, \tau_{N}}-\bar{X}_{1, \tau_{N}}\right)\right) \\
& <-(N-2) \gamma,
\end{aligned}
$$

where we note that $\mathbf{X}_{0}=\left(\frac{1}{N}, 0,0\right), \mathbf{X}_{\tau_{N}}=\left(1-\frac{1}{N}, \bar{X}_{0, \tau_{N}}, \bar{X}_{1, \tau_{N}}\right)$ and $\bar{X}_{0, \tau_{N}}<\bar{X}_{1, \tau_{N}}$ by the definition of $\tau_{N}$ and $\delta_{e}$. So we have

$$
\begin{aligned}
\lim _{N \rightarrow \infty} \frac{1}{N} \log \mathbb{E}[L(\mathcal{P}) I(\mathcal{P})] & =\lim _{N \rightarrow \infty} \frac{1}{N} \log (\mathbb{E}[L(\mathcal{P}) I(\mathcal{P}) \mid I(\mathcal{P})=1] \mathbb{P}(I(\mathcal{P})=1)) \\
& \leq \lim _{N \rightarrow \infty} \frac{1}{N} \log \left(\mathbb{E}\left[e^{-(N-2) \gamma}\right] p_{N}\right) \\
& =-2 \gamma,
\end{aligned}
$$

where the inequality follows from (11) and by the definition of $p_{N}$, and the second equality follows by using (4).

Remark 8 In the proof of Theorem 1, we see why we defined $\delta_{e}$ as in (3), and not simply $\delta_{e}=\left\{\mathbf{x}: x_{1}=1\right\}$; this allows for the second (strict) inequality to hold.

\subsection{The two-node $G|| G|| 1$ tandem queue}

Although the subsolution approach for the single queue results in a state-independent and asymptotically efficient change of measure, it is known from both [2] (general distributions) and [3,10] (Markovian distribution) that a state-independent change of measure for the $G I|G I| 1$ tandem queue cannot be asymptotically efficient for all input 
parameters. We will see that the subsolution method, as it did for the Markovian case in $[6,8]$, results in a state-dependent change of measure.

In this section, we will use the same approach as in Sect. 3.1 and we conclude by proving asymptotic efficiency of the constructed change of measure.

\subsubsection{Solutions to $\mathbb{H}(\mathrm{x}, \alpha) \geq 0$ for all possible $\mathrm{x}$}

We start the method by finding a solution to $\mathbb{H}(\mathbf{x}, \boldsymbol{\alpha}) \geq 0$ for all possible $\mathbf{x}$. As before, we let $\operatorname{DW}(\mathbf{x})=\left(\alpha_{1}, \alpha_{2}, \bar{\alpha}_{0}, \bar{\alpha}_{1}, \bar{\alpha}_{2}\right)$ and recall that $\mathcal{X}(\mathbf{x})=\min _{k \in\{0\} \cup\left\{j: x_{j}>0\right\}}\left\{\bar{x}_{k}\right\}$. Then, we have from (6), for all possible $\mathbf{x} \neq\left(\frac{1}{N}, 0,0,0,0\right)$,

$$
\begin{aligned}
\mathbb{H}(\mathbf{x}, \boldsymbol{\alpha})= & -\mathcal{X}(\mathbf{x}) N\left(\bar{\alpha}_{0}+\bar{\alpha}_{1} \mathbb{1}\left\{x_{1}>0\right\}+\bar{\alpha}_{2} \mathbb{1}\left\{x_{2}>0\right\}\right) \\
& + \begin{cases}-\log \left(e^{-\alpha_{1}} M_{A}\left(-\bar{\alpha}_{0}\right) M_{B^{(1)}}\left(-\bar{\alpha}_{1} \mathbb{1}\left\{x_{1}=0\right\}\right)\right) & \text { if } \mathcal{X}(\mathbf{x})=\bar{x}_{0}, \\
-\log \left(e^{\alpha_{1}-\alpha_{2}} M_{B^{(1)}}\left(-\bar{\alpha}_{1} \mathbb{1}\left\{x_{1}>\frac{1}{N}\right\}\right) M_{B^{(2)}}\left(-\bar{\alpha}_{2} \mathbb{1}\left\{x_{2}=0\right\}\right)\right) & \text { if } \mathcal{X}(\mathbf{x})=\bar{x}_{1}, \\
-\log \left(e^{\alpha_{2}} M_{B^{(2)}}\left(-\bar{\alpha}_{2} \mathbb{1}\left\{x_{2}>\frac{1}{N}\right\}\right)\right) & \text { if } \mathcal{X}(\mathbf{x})=\bar{x}_{2},\end{cases} \\
= & -\mathcal{X}(\mathbf{x}) N\left(\bar{\alpha}_{0}+\bar{\alpha}_{1} \mathbb{1}\left\{x_{1}>0\right\}+\bar{\alpha}_{2} \mathbb{1}\left\{x_{2}>0\right\}\right) \\
& + \begin{cases}\alpha_{1}-\Lambda_{A}\left(-\bar{\alpha}_{0}\right)-\mathbb{1}\left\{x_{1}=0\right\} \Lambda_{B^{(1)}}\left(-\bar{\alpha}_{1}\right) & \text { if } \mathcal{X}(\mathbf{x})=\bar{x}_{0}, \\
-\alpha_{1}+\alpha_{2}-\mathbb{1}\left\{x_{1}>\frac{1}{N}\right\} \Lambda_{B^{(1)}}\left(-\bar{\alpha}_{1}\right) & \text { if } \mathcal{X}(\mathbf{x})=\bar{x}_{1}, \\
-\mathbb{1}\left\{x_{2}=0\right\} \Lambda_{B^{(2)}}\left(-\bar{\alpha}_{2}\right) & \text { if } \mathcal{X}(\mathbf{x})=\bar{x}_{2}, \\
-\alpha_{2}-\mathbb{1}\left\{x_{2}>\frac{1}{N}\right\} \Lambda_{B^{(2)}}\left(-\bar{\alpha}_{2}\right) & \end{cases}
\end{aligned}
$$

and

$$
\mathbb{H}\left(\left(\frac{1}{N}, 0,0,0,0\right), \boldsymbol{\alpha}\right)=-\Lambda_{A}\left(-\bar{\alpha}_{0}\right)-\Lambda_{B^{(1)}}\left(-\bar{\alpha}_{1}\right) .
$$

The solutions to $\mathbb{H}(\mathbf{x}, \boldsymbol{\alpha}) \geq 0$ that we describe depend on the number of customers in queue 1 and the number of customers in queue 2 . Note that there may be more solutions possible, but in order to find a state-dependent change of measure the current solutions turn out to be sufficient. Clearly, $\boldsymbol{\alpha}=\mathbf{0}$ is always a solution to $\mathbb{H}(\mathbf{x}, \boldsymbol{\alpha}) \geq 0$ and is equivalent to no change of measure. However, using $\operatorname{DW}(\mathbf{x})=\boldsymbol{\alpha}=\mathbf{0}$ does not give a subsolution, since in that case it is impossible to satisfy properties 3 and 4 in Definition 2.

In Table $1, \widetilde{\theta}^{(1)}=\sup \left\{\theta: \Lambda_{A}\left(-\theta^{*}\right)+\Lambda_{B^{(1)}}(\theta) \leq 0\right\}$ and $\widetilde{\theta}^{(2)}=\sup \{\theta$ : $\left.\Lambda_{A}\left(-\theta^{*}\right)+\Lambda_{B^{(2)}}(\theta) \leq 0\right\}$; see Figs. 3 and 4 for a graphical illustration of $\theta_{1}, \theta_{2}, \widetilde{\theta}^{(1)}$

Table 1 Some solutions to $\mathbb{H}(\mathbf{x}, \boldsymbol{\alpha}) \geq 0$ for all possible $\mathbf{x}$

\begin{tabular}{llllll}
\hline Region of validity & $\alpha_{1}$ & $\alpha_{2}$ & $\bar{\alpha}_{0}$ & $\bar{\alpha}_{1}$ & $\bar{\alpha}_{2}$ \\
\hline$x_{1}>0, x_{2}>0$ & $\Lambda_{A}\left(-\theta^{*}\right)$ & 0 & $\theta^{*}$ & {$\left[-\widetilde{\theta}^{(1)},-\theta^{*}\right]$} & {$\left[0,-\bar{\alpha}_{1}-\theta^{*}\right]$} \\
& $\Lambda_{A}\left(-\theta^{*}\right)$ & $\Lambda_{A}\left(-\theta^{*}\right)$ & $\theta^{*}$ & {$\left[0,-\bar{\alpha}_{2}-\theta^{*}\right]$} & {$\left[-\widetilde{\theta}^{(2)},-\theta^{*}\right]$} \\
$x_{1}>0, x_{2}=0$ & $\Lambda_{A}\left(-\theta^{*}\right)$ & 0 & $\theta^{*}$ & {$\left[-\widetilde{\theta}^{(1)},-\theta^{*}\right]$} & {$\left[0,-\bar{\alpha}_{1}-\theta^{*}\right]$} \\
$x_{1}=0, x_{2}>0$ & $\Lambda_{A}\left(-\theta^{*}\right)$ & $\Lambda_{A}\left(-\theta^{*}\right)$ & $\theta^{*}$ & {$\left[0,-\bar{\alpha}_{2}-\theta^{*}\right]$} & {$\left[-\widetilde{\theta}^{(2)},-\theta^{*}\right]$} \\
$x_{1} \geq 0, x_{2} \geq 0$ & 0 & 0 & 0 & 0 & 0 \\
\hline
\end{tabular}




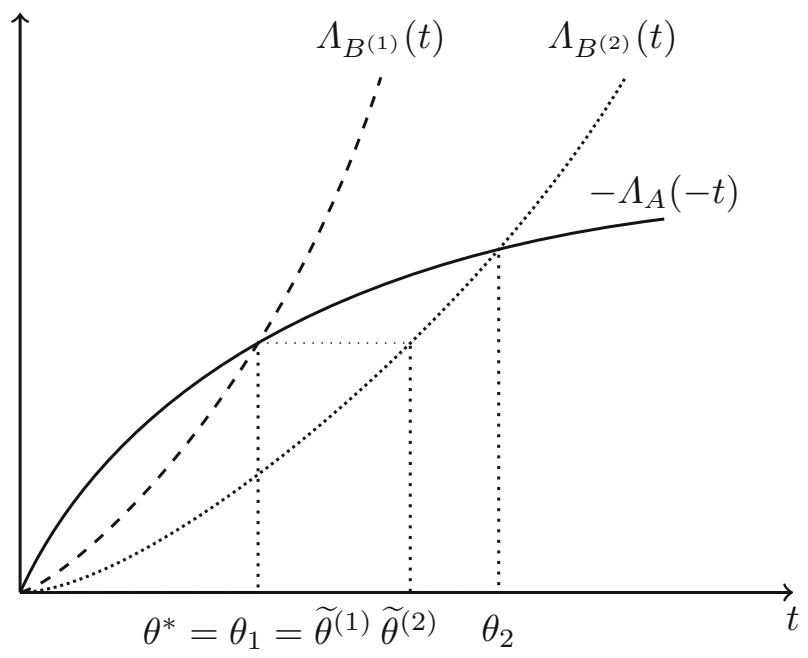

Fig. 3 Illustration of $\theta_{1}, \theta_{2}, \widetilde{\theta}^{(1)}$ and $\widetilde{\theta}^{(2)}$ when queue 1 is the bottleneck queue

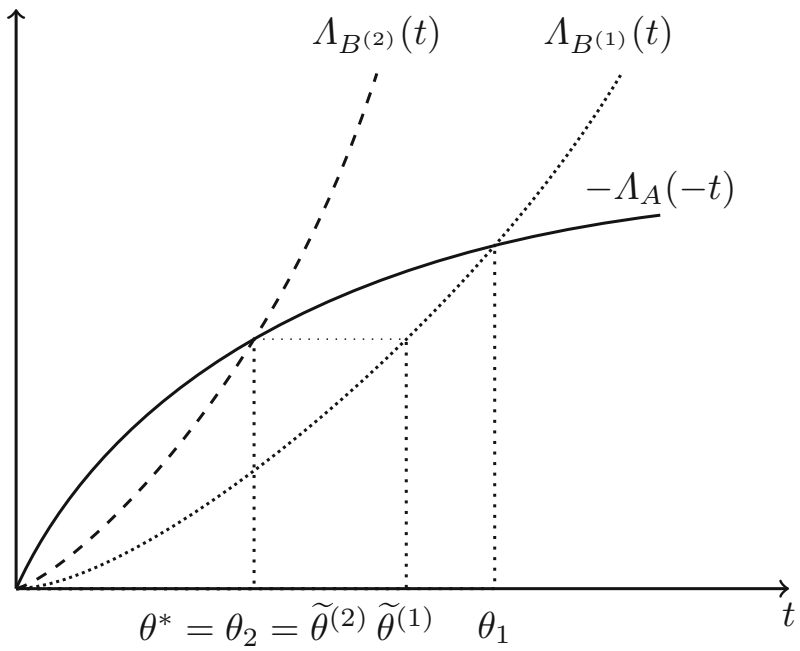

Fig. 4 Similar to Fig. 3, but when queue 2 is the bottleneck queue

and $\widetilde{\theta}^{(2)}$. Note that for all solutions proposed in Table 1 we have

$$
\mathbb{H}\left(\left(\frac{1}{N}, 0,0,0,0\right), \boldsymbol{\alpha}\right)=-\Lambda_{A}\left(-\bar{\alpha}_{0}\right)-\Lambda_{B^{(1)}}\left(-\bar{\alpha}_{1}\right) \geq 0
$$

Remark 9 All solutions presented in Table 1 satisfy subsolution property 2 along the most likely path (as defined below Definition 1) with equality. Recall that, along the most likely path, $x_{j}>0$ when queue $j$ is the bottleneck queue. Note that the only solutions that do not necessarily satisfy $\mathbb{H}(\mathbf{x}, \boldsymbol{\alpha})=0$ are those along the boundary 
$x_{j}=0$ when queue $j$ is the bottleneck queue (except for $\boldsymbol{\alpha}=\mathbf{0}$, which is always possible).

Remark 10 We see from Table 1 that in some cases there is a possibility to choose $\bar{\alpha}_{j}>$ 0 . It is not obvious to do so, because then the service times of queue $j$ are tilted in the 'wrong' way, since it would imply that on average the service times of queue $j$ are even shorter than under the original measure. Nevertheless, it is a solution that satisfies $\mathbb{H}(\mathbf{x}, \boldsymbol{\alpha}) \geq 0$.

\subsubsection{Construction of $W(\mathrm{x})$}

Since there is no non-trivial solution for $\mathbb{H}(\mathbf{x}, \boldsymbol{\alpha}) \geq 0$ that holds for all $\mathbf{x}$, that is, there is not a single affine function $W(\mathbf{x})$ such that for its derivative $\boldsymbol{\alpha}$ this condition holds for all $\mathbf{x}$, it turns out that a different change of measure needs to be applied in different 'regions' of the state space that are specified precisely later.

From Table 1, we can extract two non-trivial solutions that are dependent on the bottleneck queue: one which we can use for $x_{1}>0$ (and any $x_{2}$ ) and one which we can use for $x_{2}>0$ (and any $x_{1}$ ). These solutions, along with the trivial solution, can be found in Table 2 and are sufficient to obtain an asymptotically efficient estimator (as we will prove in Sect. 3.2.4; see Theorem 2). In the construction that is explained below, we will also see that cases in which both $x_{j}>0$ can be handled using these subsolutions; see Eq. (14). As it turns out, when both $x_{j}>0$, either the solution for $x_{1}>0$ or the solution for $x_{2}>0$ will be used.

Remark 11 The other solutions that are described in Table 1 can also be used to find a state-dependent change of measure that is proven to be asymptotically efficient using the same method that is described below; see Theorem 3.

Remember that $\gamma=-\Lambda_{A}\left(-\theta^{*}\right)$, and so we define

$$
\begin{aligned}
& \boldsymbol{\alpha}_{1}=\left(-\gamma,-\gamma, \theta^{*}, 0,-\theta^{*}\right), \\
& \boldsymbol{\alpha}_{2}=\left(-\gamma, 0, \theta^{*},-\theta^{*}, 0\right), \\
& \boldsymbol{\alpha}_{3}=(0,0,0,0,0),
\end{aligned}
$$

which are used to determine the different changes of measure in each of the 'regions' of the state space. Thus, 'region' 1 -corresponding to $\alpha_{1}$-has to cover $x_{1}=0$, 'region' 2-corresponding to $\boldsymbol{\alpha}_{2}$-has to cover $x_{2}=0$ and, finally, 'region' 3corresponding to $\boldsymbol{\alpha}_{3}$ - has to cover $x_{1}=x_{2}=0$. Note that $\boldsymbol{\alpha}_{k}$ is the notation for a

Table 2 Solutions extracted from Table 1 that are used for the state-dependent change of measure

\begin{tabular}{llllll}
\hline Region of validity & $\alpha_{1}$ & $\alpha_{2}$ & $\bar{\alpha}_{0}$ & $\bar{\alpha}_{1}$ & $\bar{\alpha}_{2}$ \\
\hline$x_{2}>0$ & $\Lambda_{A}\left(-\theta^{*}\right)$ & $\Lambda_{A}\left(-\theta^{*}\right)$ & $\theta^{*}$ & 0 & $-\theta^{*}$ \\
$x_{1}>0$ & $\Lambda_{A}\left(-\theta^{*}\right)$ & 0 & $\theta^{*}$ & $-\theta^{*}$ & 0 \\
$x_{1} \geq 0, x_{2} \geq 0$ & 0 & 0 & 0 & 0 & 0 \\
\hline
\end{tabular}


solution to $\mathbb{H}(\mathbf{x}, \boldsymbol{\alpha}) \geq 0$, whereas $\alpha_{k}$ is the notation for a component of some vector $\boldsymbol{\alpha}$. From these solutions $\boldsymbol{\alpha}_{k}$, we define three functions

$$
W_{k}^{\delta}(\mathbf{x})=\left\langle\boldsymbol{\alpha}_{k}, \mathbf{x}\right\rangle+\gamma-k \delta, \quad \text { for } k=1,2,3,
$$

for some $\delta>0$, and their minimum

$$
W^{\delta}(\mathbf{x})=W_{1}^{\delta}(\mathbf{x}) \wedge W_{2}^{\delta}(\mathbf{x}) \wedge W_{3}^{\delta}(\mathbf{x}) .
$$

The idea of this construction, as in [6-8], and in particular subtracting $k \delta$, is that the minimum function $W^{\delta}(\mathbf{x})$ has gradient $\boldsymbol{\alpha}_{1}$ near $x_{1}=0$, gradient $\boldsymbol{\alpha}_{2}$ near $x_{2}=0$ and gradient $\boldsymbol{\alpha}_{3}$ near $x_{1}=x_{2}=0$, as desired.

In Figs. 5 and 6, we give a rough illustration of the behaviour of the function $W^{\delta}(\mathbf{x})$, considering the dependence on the scaled queue lengths $x_{1}$ and $x_{2}$ while neglecting the dependence on the scaled residuals $\bar{x}_{0}, \bar{x}_{1}$ and $\bar{x}_{2}$. For large $N$, these can indeed be neglected due to scaling, in particular in the case of bounded supports of the inter-arrival time and service times. However, for unbounded supports there can be exceptions to this idea due to a very large residual inter-arrival time or a very large residual service time. For example, at $x_{1}=0$ we have $W_{3}^{\delta}(\mathbf{x})<\min \left\{W_{1}^{\delta}(\mathbf{x}), W_{2}^{\delta}(\mathbf{x})\right\}$ if and only if $x_{2}<\frac{2 \delta+\theta^{*}\left(\bar{x}_{0}-\bar{x}_{2}\right)}{\gamma}$. When $\bar{x}_{0}-\bar{x}_{2}$ is large, $W_{3}^{\delta}(\mathbf{x})$ is the minimum function even when $x_{2}$ is not near 0 . Indeed, in this case it is very likely that the system empties before the next arriving customer, and hence it makes sense that $W^{\delta}(\mathbf{x})=W_{3}^{\delta}(\mathbf{x})$ (and no change of measure will be applied).

Note that $W^{\delta}(\mathbf{x})$ satisfies properties $2-4$ in and below Definition 2 by construction. As we have different functions for different regions, $W^{\delta}(\mathbf{x})$ is not a continuously differentiable function, which is the first property of a classical subsolution. Therefore, we apply a similar mollification procedure as in previous work on Markovian systems;

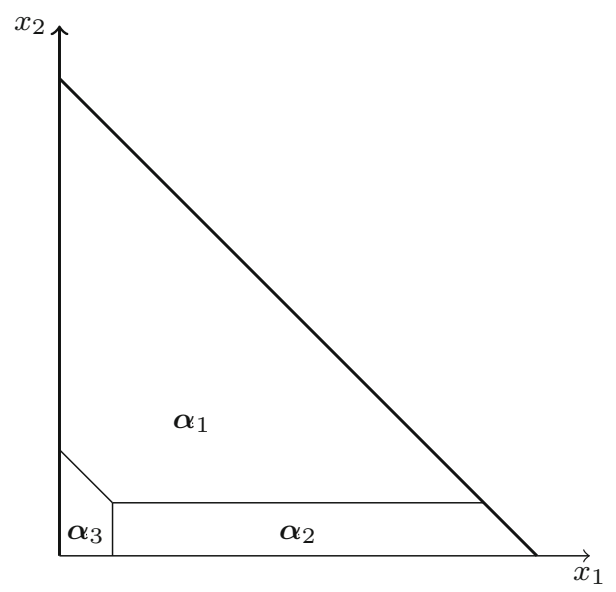

Fig. 5 Main dimensions $\left(x_{1}, x_{2}\right)$ (the scaled queue sizes) of the state space, neglecting $\bar{x}_{0}, \bar{x}_{1}, \bar{x}_{2}$ (the scaled residuals) by setting them equal to 0 . The figure shows the three regions where the different $W_{k}^{\delta}(\mathbf{x})$, $k=1,2,3$, are the minimum function 


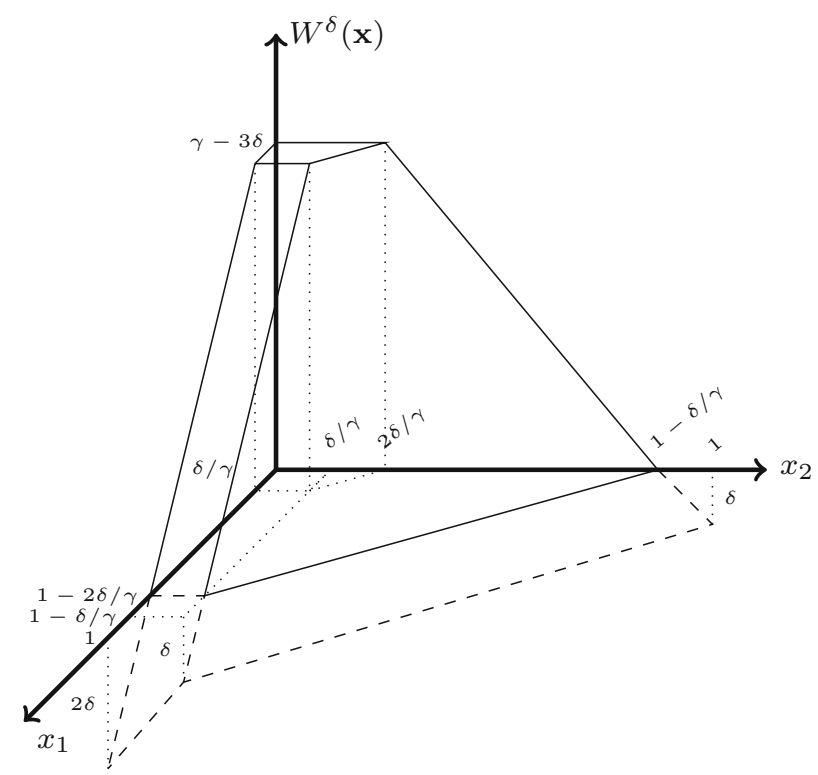

Fig. 6 Illustration of the dependence of $W^{\delta}(\mathbf{x})$ on $\left(x_{1}, x_{2}\right)$, neglecting the scaled residuals as in Fig. 5. The figure here shows how for $\mathbf{x}$ in region $k$ we have $W^{\delta}(\mathbf{x})=W_{k}^{\delta}(\mathbf{x})$, and hence $\mathrm{DW}^{\delta}(\mathbf{x})=\boldsymbol{\alpha}_{k}$

see $[6,8]$. This mollification ensures that a derivative exists throughout the whole parameter space. We let

$$
W^{\varepsilon, \delta}(\mathbf{x})=-\varepsilon \log \sum_{k=1}^{3} e^{-W_{k}^{\delta}(\mathbf{x}) / \varepsilon} .
$$

When $\varepsilon \rightarrow 0, W^{\varepsilon, \delta}(\mathbf{x})$ converges to $W^{\delta}(\mathbf{x})$. Another result of this choice of $W^{\varepsilon, \delta}(\mathbf{x})$ is that

$$
\begin{aligned}
\mathrm{DW}^{\varepsilon, \delta}(\mathbf{x})= & \sum_{k=1}^{3} \rho_{k}(\mathbf{x}) \boldsymbol{\alpha}_{k} \\
= & \left(-\left(\rho_{1}(\mathbf{x})+\rho_{2}(\mathbf{x})\right) \gamma,-\rho_{1}(\mathbf{x}) \gamma,\left(\rho_{1}(\mathbf{x})+\rho_{2}(\mathbf{x})\right) \theta^{*},\right. \\
& \left.-\rho_{2}(\mathbf{x}) \theta^{*},-\rho_{1}(\mathbf{x}) \theta^{*}\right),
\end{aligned}
$$

with

$$
\rho_{k}(\mathbf{x})=\frac{e^{-W_{k}^{\delta}(\mathbf{x}) / \varepsilon}}{\sum_{j} e^{-W_{j}^{\delta}(\mathbf{x}) / \varepsilon}} .
$$

Throughout this paper, we make the following assumptions on $\varepsilon$ and $\delta$, as in [6]. We remark that we let $\varepsilon$ and $\delta$ depend on $N$, though for brevity we do not explicitly write this dependence.

Assumption 3 We choose $\varepsilon$ and $\delta$ dependent on $N$, such that

- $\lim _{N \rightarrow \infty} \varepsilon=0$, 
- $\lim _{N \rightarrow \infty} \delta=0$,

- $\lim _{N \rightarrow \infty} \varepsilon N=\infty$,

- $\lim _{N \rightarrow \infty} \frac{\varepsilon}{\delta}=0$.

As a result, $W^{\varepsilon, \delta}(\mathbf{x})$ satisfies properties 3 and 4 in and below Definition 2 as $N \rightarrow \infty$, which follows immediately from the following lemma.

Lemma 1 Under Assumption 1, we have, for the two-node GI $|G I| 1$ tandem queue, that $W^{\varepsilon, \delta}(\mathbf{x}) \leq \frac{\gamma}{N}-\delta$ for $\mathbf{x} \in \delta_{e}$ and $\gamma\left(1-\frac{1}{N}\right)-3 \delta-\varepsilon \log (3) \leq W^{\varepsilon, \delta}\left(\mathbf{X}_{0}\right) \leq \gamma-3 \delta$.

Proof We have, for $\mathbf{x} \in \delta_{e}$, by (15),

$$
W^{\varepsilon, \delta}(\mathbf{x}) \leq W_{1}^{\delta}(\mathbf{x})=-\gamma\left(x_{1}+x_{2}\right)+\theta^{*}\left(\bar{x}_{0}-\bar{x}_{2}\right)+\gamma-\delta \leq \frac{\gamma}{N}-\delta,
$$

where the final inequality follows as $x_{1}+x_{2}=1-\frac{1}{N}$ and $\bar{x}_{0}-\bar{x}_{2} \leq 0$ for $\mathbf{x} \in \delta_{e}$.

For $W^{\varepsilon, \delta}\left(\mathbf{X}_{0}\right)$, we note that $\mathbf{X}_{0}=\left(\frac{1}{N}, 0,0,0,0\right)$. By (15), we have

$$
\begin{aligned}
W^{\varepsilon, \delta}\left(\mathbf{X}_{0}\right) & \leq W_{3}^{\delta}\left(\mathbf{X}_{0}\right)=\gamma-3 \delta \\
W^{\varepsilon, \delta}\left(\mathbf{X}_{0}\right) & =-\varepsilon \log \left(e^{\left(-\gamma\left(1-\frac{1}{N}\right)+\delta\right) / \varepsilon}+e^{\left(-\gamma\left(1-\frac{1}{N}\right)+2 \delta\right) / \varepsilon}+e^{(-\gamma+3 \delta) / \varepsilon}\right) \\
& \geq-\varepsilon \log \left(3 e^{\left(-\gamma+\frac{\gamma}{N}+3 \delta\right) / \varepsilon}\right) \\
& =\gamma\left(1-\frac{1}{N}\right)-3 \delta-\varepsilon \log (3) .
\end{aligned}
$$

Remark 12 The proof of Lemma 1 again explains our choice of $\delta_{e}$ : it is chosen such that $W^{\varepsilon, \delta}(\mathbf{x})$ can be upper bounded for $\mathbf{x} \in \delta_{e}$.

Note that, by construction, we expect that also the second property of a classical subsolution is satisfied for $W^{\varepsilon, \delta}(\mathbf{x})$, i.e., $\mathbb{H}\left(\mathbf{x}, \mathrm{DW}^{\varepsilon, \delta}(\mathbf{x})\right) \geq 0$ when $N \rightarrow \infty$, which we will prove in Lemma 2.

\subsubsection{The change of measure}

In this section, we will give examples of the change of measure based on $W_{k}^{\delta}(\mathbf{x})$, see (13), for some parts of the state description. This gives some insight into the change of measure that is applied in the mollified function $W^{\varepsilon, \delta}(\mathbf{x})$ and how the change of measure that we use in the current paper relates to previous work. When choosing $\mathrm{DW}_{k}^{\delta}(\mathbf{x})$ in (7) as a constant vector $\boldsymbol{\alpha}=\left(\alpha_{1}, \alpha_{2}, \bar{\alpha}_{0}, \bar{\alpha}_{1}, \bar{\alpha}_{2}\right)$, the change of measure can be written in terms of $\bar{\alpha}_{0}, \bar{\alpha}_{1}$ and $\bar{\alpha}_{2}$ as 


$$
\begin{aligned}
& \mathrm{d} \bar{F}_{V_{X}(\mathbf{x})}(\mathbf{v} \mid \mathbf{x})=
\end{aligned}
$$

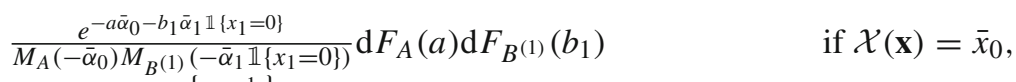

$$
\begin{aligned}
& \frac{e^{-b_{1} \bar{\alpha}_{1} \mathbb{1}\left\{x_{1}>\frac{1}{N}\right\}-b_{2} \bar{\alpha}_{2} \mathbb{1}\left\{x_{2}=0\right\}}}{M_{B^{(1)}}\left(-\bar{\alpha}_{1} \mathbb{1}\left\{x_{1}>\frac{1}{N}\right\}\right) M_{B^{(2)}}\left(-\bar{\alpha}_{2} \mathbb{1}\left\{x_{2}=0\right\}\right)} \mathrm{d} F_{B^{(1)}}\left(b_{1}\right) \mathrm{d} F_{B^{(2)}}\left(b_{2}\right) \text { if } \mathcal{X}(\mathbf{x})=\bar{x}_{1}, \\
& \frac{e^{-b_{2} \bar{\alpha}_{2} \mathbb{1}\left\{x_{2}>\frac{1}{N}\right\}}}{M_{B}(2)\left(-\bar{\alpha}_{2} \mathbb{1}\left\{x_{2}>\frac{1}{N}\right\}\right)} \mathrm{d} F_{B^{(2)}}\left(b_{2}\right) \quad \text { if } \mathcal{X}(\mathbf{x})=\bar{x}_{2},
\end{aligned}
$$

for $\mathbf{x} \neq \mathbf{X}_{0}$ and

$$
\mathrm{d} \bar{F}_{V_{X}\left(\mathbf{X}_{0}\right)}\left(\mathbf{v} \mid \mathbf{X}_{0}\right)=\frac{e^{-a \bar{\alpha}_{0}-b_{1} \bar{\alpha}_{1}}}{M_{A}\left(-\bar{\alpha}_{0}\right) M_{B^{(1)}}\left(-\bar{\alpha}_{1}\right)} \mathrm{d} F_{A}(a) \mathrm{d} F_{B^{(1)}}\left(b_{1}\right) .
$$

In each of the examples below, we consider the cases where our change of measure based on $W^{\varepsilon, \delta}(\mathbf{x})$ gets close, as $N \rightarrow \infty$, to an 'affine' change of measure as above, that is, based on some $W_{k}^{\delta}(\mathbf{x})$ with constant gradient $\boldsymbol{\alpha}$. It is two of these latter 'limiting' change of measures that we consider in the following.

\section{Examples of the change of measure}

Example 1 Where $W^{\varepsilon, \delta}(\mathbf{x})$ gets close to $W_{2}^{\delta}(\mathbf{x})$, we let $\alpha=\mathrm{DW}_{2}^{\delta}(\mathbf{x})=\left(-\gamma, 0, \theta^{*}\right.$, $\left.-\theta^{*}, 0\right)$. Then (18) and (19) reduce to

$$
\begin{aligned}
& \mathrm{d} \bar{F}_{V_{X}(\mathbf{x})}(\mathbf{v} \mid \mathbf{x})
\end{aligned}
$$

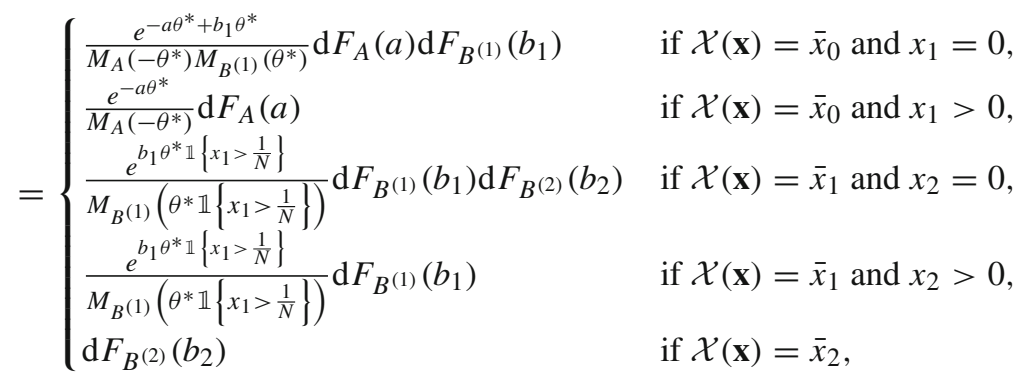

for $\mathbf{x} \neq \mathbf{X}_{0}$ and

$$
\mathrm{d} \bar{F}_{V_{X}\left(\mathbf{X}_{0}\right)}\left(\mathbf{v} \mid \mathbf{X}_{0}\right)=\frac{e^{-a \theta^{*}+b_{1} \theta^{*}}}{M_{A}\left(-\theta^{*}\right) M_{B^{(1)}}\left(\theta^{*}\right)} \mathrm{d} F_{A}(a) \mathrm{d} F_{B^{(1)}}\left(b_{1}\right) .
$$

When queue 1 is the bottleneck queue, this corresponds to the state-independent change of measure that has been studied in $[2,9,11]$. On the other hand, when queue 2 is the bottleneck queue, the inter-arrival times are still exponentially tilted with parameter $-\theta^{*}=-\theta_{2}$ and so it also corresponds to the state-independent change of measure studied in those papers. However, it is not the service times of queue 2 that are exponentially tilted, but the service times of queue 1 that are exponentially tilted with parameter $\theta^{*}=\theta_{2}$. 
Remark 13 We note that the change of measure used here along the horizontal boundary is different from the one in [6] for a two-node Markovian tandem queue. Let $\lambda, \mu_{1}$ and $\mu_{2}$ be the exponential rates for the inter-arrival times, and service times of queue 1 and queue 2 under the original measure. If we would consider $\mathrm{DW}_{2}^{\delta}(\mathbf{x}) \equiv \boldsymbol{\alpha}_{2}=\left(-\gamma, 0, \theta^{*},-\widetilde{\theta}^{(1)}, 0\right)$, which is a solution to $\mathbb{H}(\mathbf{x}, \boldsymbol{\alpha}) \geq 0$ according to Table 1 , then we find that under the change of measure the service times of queue 1 have an exponential distribution with rate $\frac{\mu_{1} \lambda}{\mu_{2}}$. This does correspond with the results from [6], although they consider the embedded discrete-time Markov chain, while we consider the continuous-time Markov chain.

Example 2 Where $W^{\varepsilon, \delta}(\mathbf{x})$ gets close to $W_{1}^{\delta}(\mathbf{x})$, we let $\alpha=\mathrm{DW}_{1}^{\delta}(\mathbf{x})=(-\gamma$, $\left.-\gamma, \theta^{*}, 0,-\theta^{*}\right)$. Then, (18) and (19) reduce to

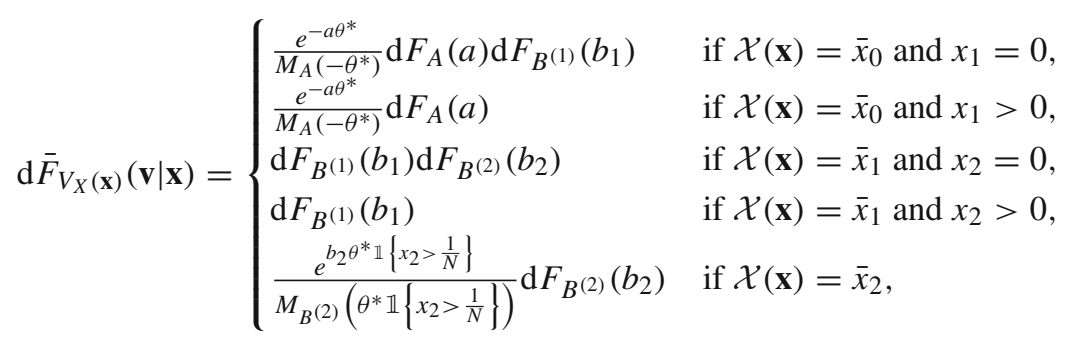

for $\mathbf{x} \neq \mathbf{X}_{0}$ and

$$
\mathrm{d} \bar{F}_{V_{X}\left(\mathbf{X}_{0}\right)}\left(\mathbf{v} \mid \mathbf{X}_{0}\right)=\frac{e^{-a \theta^{*}}}{M_{A}\left(-\theta^{*}\right)} \mathrm{d} F_{A}(a) \mathrm{d} F_{B^{(1)}}\left(b_{1}\right) .
$$

When queue 1 is the bottleneck queue, this change of measure corresponds to the stateindependent change of measure studied in $[2,9,11]$ in the sense that the inter-arrival times are exponentially tilted with parameter $-\theta^{*}=-\theta_{1}$. In contrast to an exponential tilt for the service times of queue 1 , which happens for the state-independent change of measure, here we exponentially tilt the service times of queue 2 with parameter $\theta^{*}=\theta_{1}$. When queue 2 is the bottleneck queue, the change of measure described above corresponds to the state-independent change of measure that has been studied in $[2,9,11]$.

\subsubsection{Asymptotic efficiency}

Using (16), we can find a lower bound on $\mathbb{H}\left(\mathbf{x}, \mathrm{DW}^{\varepsilon, \delta}(\mathbf{x})\right)$ which goes to 0 when $N \rightarrow \infty$.

Remark 14 Since we assume bounded supports of the various service time distributions, we note that equality is achieved in (5) for all queues $j$. That is, we have $\Lambda_{A}\left(-\theta^{(j)}\right)+\Lambda_{B^{(j)}}\left(\theta^{(j)}\right)=0$ for all queues $j$. In particular, equality holds for the bottleneck queue. Similarly, equality in the definition of $\widetilde{\theta}^{(1)}$ and $\widetilde{\theta}^{(2)}$ also holds. 
Lemma 2 Under Assumptions 1 and 2, we have, for the two-node GI|GI|1 tandem queue, for all possible $\mathbf{x}$,

$$
\mathbb{H}\left(\mathbf{x}, D W^{\varepsilon, \delta}(\mathbf{x})\right) \geq-\left(\theta^{*} \max \left\{Q^{(0)}, Q^{(1)}, Q^{(2)}\right\}+\gamma\right) e^{-\delta / \varepsilon} .
$$

Proof We substitute (16) in (12) to find $\mathbb{H}\left(\mathbf{x}, \mathrm{DW}^{\varepsilon, \delta}(\mathbf{x})\right)$ for $\mathbf{x} \neq \mathbf{X}_{0}$ equal to

$$
\begin{aligned}
& -\mathcal{X}(\mathbf{x}) N\left(\left(\rho_{1}(\mathbf{x})+\rho_{2}(\mathbf{x})\right) \theta^{*}-\rho_{2}(\mathbf{x}) \theta^{*} \mathbb{1}\left\{x_{1}>0\right\}-\rho_{1}(\mathbf{x}) \theta^{*} \mathbb{1}\left\{x_{2}>0\right\}\right) \\
& + \begin{cases}-\left(\rho_{1}(\mathbf{x})+\rho_{2}(\mathbf{x})\right) \gamma-\Lambda_{A}\left(-\left(\rho_{1}(\mathbf{x})+\rho_{2}(\mathbf{x})\right) \theta^{*}\right) & \text { if } \mathcal{X}(\mathbf{x})=\bar{x}_{0}, \\
-\mathbb{1}\left\{x_{1}=0\right\} \Lambda_{B^{(1)}}\left(\rho_{2}(\mathbf{x}) \theta^{*}\right) & \text { if } \mathcal{X}(\mathbf{x})=\bar{x}_{1}, \\
\left(\rho_{1}(\mathbf{x})+\rho_{2}(\mathbf{x})\right) \gamma-\rho_{1}(\mathbf{x}) \gamma-\mathbb{1}\left\{x_{1}>\frac{1}{N}\right\} \Lambda_{B^{(1)}}\left(\rho_{2}(\mathbf{x}) \theta^{*}\right) & \text { if } \mathcal{X}(\mathbf{x})=\bar{x}_{2}, \\
-\mathbb{1}\left\{x_{2}=0\right\} \Lambda_{B^{(2)}}\left(\rho_{1}(\mathbf{x}) \theta^{*}\right) & \text { if } \mathcal{X}(\mathbf{x})=\bar{x}_{0}, \\
\rho_{1}(\mathbf{x}) \gamma-\mathbb{1}\left\{x_{2}>\frac{1}{N}\right\} \Lambda_{B^{(2)}}\left(\rho_{1}(\mathbf{x}) \theta^{*}\right) & \text { if } \mathcal{X}(\mathbf{x})=\bar{x}_{1}, \\
\geq-\mathcal{X}(\mathbf{x}) N\left(\rho_{1}(\mathbf{x}) \theta^{*} \mathbb{1}\left\{x_{2}=0\right\}+\rho_{2}(\mathbf{x}) \theta^{*} \mathbb{1}\left\{x_{1}=0\right\}\right) & \text { if } \mathcal{X}(\mathbf{x})=\bar{x}_{2},\end{cases} \\
& + \begin{cases}-\rho_{2}(\mathbf{x}) \mathbb{1}\left\{x_{1}=0\right\} \Lambda_{B^{(1)}}\left(\theta^{*}\right) & \rho_{2}(\mathbf{x}) \gamma-\rho_{2}(\mathbf{x}) \mathbb{1}\left\{x_{1}>\frac{1}{N}\right\} \Lambda_{B^{(1)}}\left(\theta^{*}\right) \\
-\rho_{1}(\mathbf{x}) \mathbb{1}\left\{x_{2}=0\right\} \Lambda_{B^{(2)}}\left(\theta^{*}\right) & \rho_{1}(\mathbf{x}) \mathbb{1}\left\{x_{2}>\frac{1}{N}\right\} \Lambda_{B^{(2)}}\left(\theta^{*}\right)\end{cases}
\end{aligned}
$$

where the inequality follows from convexity of the log-moment generating functions, $\rho_{k}(\mathbf{x}) \in[0,1]$ and by the definition of $\gamma=-\Lambda_{A}\left(-\theta^{*}\right) \geq 0$. By using $\mathbb{1}\left\{x_{k}>\frac{1}{N}\right\} \leq 1$ for $k=1,2, \Lambda_{A}\left(-\theta^{*}\right)+\Lambda_{B^{(j)}}\left(\theta^{*}\right) \leq 0$ for all queues $j$ by the definition of $\theta^{*}, \gamma$ and the bounded supports (and hence bounded $\mathcal{X}(\mathbf{x})$ ), we find that (20) is greater than or equal to

$$
\begin{gathered}
-\max \left\{Q^{(0)}, Q^{(1)}, Q^{(2)}\right\}\left(\rho_{1}(\mathbf{x}) \theta^{*} \mathbb{1}\left\{x_{2}=0\right\}+\rho_{2}(\mathbf{x}) \theta^{*} \mathbb{1}\left\{x_{1}=0\right\}\right) \\
+ \begin{cases}-\rho_{2}(\mathbf{x}) \mathbb{1}\left\{x_{1}=0\right\} \Lambda_{B^{(1)}}\left(\theta^{*}\right) & \text { if } \mathcal{X}(\mathbf{x})=\bar{x}_{0}, \\
-\rho_{1}(\mathbf{x}) \mathbb{1}\left\{x_{2}=0\right\} \Lambda_{B^{(2)}}\left(\theta^{*}\right) & \text { if } \mathcal{X}(\mathbf{x})=\bar{x}_{1}, \\
0 & \text { if } \mathcal{X}(\mathbf{x})=\bar{x}_{2} .\end{cases}
\end{gathered}
$$

For any $\mathbf{x}$ with $x_{2}=\bar{x}_{2}=0$, we have, from (17),

$$
\rho_{1}(\mathbf{x})=\frac{e^{-W_{1}^{\delta}(\mathbf{x}) / \varepsilon}}{\sum_{j} e^{-W_{j}^{\delta}(\mathbf{x}) / \varepsilon}} \leq \frac{e^{-\left(\left\langle\boldsymbol{\alpha}_{1}, \mathbf{x}\right\rangle+\gamma-\delta\right) / \varepsilon}}{e^{-\left(\left\langle\boldsymbol{\alpha}_{2}, \mathbf{x}\right\rangle+\gamma-2 \delta\right) / \varepsilon}}=e^{-\left(\bar{x}_{1} \theta^{*}+\delta\right) / \varepsilon} \leq e^{-\delta / \varepsilon}
$$

and, for any $\mathbf{x}$ with $x_{1}=\bar{x}_{1}=0$,

$$
\rho_{2}(\mathbf{x})=\frac{e^{-W_{2}^{\delta}(\mathbf{x}) / \varepsilon}}{\sum_{j} e^{-W_{j}^{\delta}(\mathbf{x}) / \varepsilon}} \leq \frac{e^{-\left(\left\langle\boldsymbol{\alpha}_{2}, \mathbf{x}\right\rangle+\gamma-2 \delta\right) / \varepsilon}}{e^{-\left(\left\langle\boldsymbol{\alpha}_{3}, \mathbf{x}\right\rangle+\gamma-3 \delta\right) / \varepsilon}}=e^{-\left(\bar{x}_{0} \theta^{*}+\delta\right) / \varepsilon} \leq e^{-\delta / \varepsilon}
$$


Substituting (22) and (23) in (21), we find, for $\mathbf{x} \neq \mathbf{X}_{0}$, that

$$
\begin{aligned}
& \mathbb{H}\left(\mathbf{x}, \mathrm{DW}^{\varepsilon, \delta}(\mathbf{x})\right) \\
& \begin{aligned}
\geq & \max \left\{Q^{(0)}, Q^{(1)}, Q^{(2)}\right\}\left(\rho_{1}(\mathbf{x}) \theta^{*} \mathbb{1}\left\{x_{2}=0\right\}+\rho_{2}(\mathbf{x}) \theta^{*} \mathbb{1}\left\{x_{1}=0\right\}\right) \\
& -\rho_{2}(\mathbf{x}) \mathbb{1}\left\{x_{1}=0\right\} \Lambda_{B^{(1)}}\left(\theta^{*}\right) \mathbb{1}\left\{\mathcal{X}(\mathbf{x})=\bar{x}_{0}\right\} \\
& -\rho_{1}(\mathbf{x}) \mathbb{1}\left\{x_{2}=0\right\} \Lambda_{B^{(2)}}\left(\theta^{*}\right) \mathbb{1}\left\{\mathcal{X}(\mathbf{x})=\bar{x}_{1}\right\}
\end{aligned} \\
& \geq-e^{-\delta / \varepsilon}\left(\theta^{*} \max \left\{Q^{(0)}, Q^{(1)}, Q^{(2)}\right\}\right. \\
& \left.\quad+\Lambda_{B^{(1)}}\left(\theta^{*}\right) \mathbb{1}\left\{\mathcal{X}(\mathbf{x})=\bar{x}_{0}\right\}+\Lambda_{B^{(2)}}\left(\theta^{*}\right) \mathbb{1}\left\{\mathcal{X}(\mathbf{x})=\bar{x}_{1}\right\}\right) \\
& \geq-e^{-\delta / \varepsilon}\left(\theta^{*} \max \left\{Q^{(0)}, Q^{(1)}, Q^{(2)}\right\}+\max \left(\Lambda_{B^{(1)}}\left(\theta^{*}\right), \Lambda_{B^{(2)}}\left(\theta^{*}\right)\right)\right) \\
& =-\left(\theta^{*} \max \left\{Q^{(0)}, Q^{(1)}, Q^{(2)}\right\}+\gamma\right) e^{-\delta / \varepsilon},
\end{aligned}
$$

where the second and the third inequalities follow as at most one of the indicators equals 1 at any time during a busy cycle of the system and the final equality follows by the definition of $\theta^{*}$. To conclude the proof, we write for the initial state that

$$
\begin{aligned}
\mathbb{H}\left(\mathbf{X}_{0}, \mathrm{DW}^{\varepsilon, \delta}\left(\mathbf{X}_{0}\right)\right) & =-\Lambda_{A}\left(-\left(\rho_{1}\left(\mathbf{X}_{0}\right)+\rho_{2}\left(\mathbf{X}_{0}\right)\right) \theta^{*}\right)-\Lambda_{B^{(1)}}\left(\rho_{2}\left(\mathbf{X}_{0}\right) \theta^{*}\right) \\
& \geq-\left(\rho_{1}\left(\mathbf{X}_{0}\right)+\rho_{2}\left(\mathbf{X}_{0}\right)\right) \Lambda_{A}\left(-\theta^{*}\right)-\rho_{2}\left(\mathbf{X}_{0}\right) \Lambda_{B^{(1)}}\left(\theta^{*}\right) \geq 0
\end{aligned}
$$

Next we show that $\sum_{i=0}^{\tau_{N}-1}\left\langle\mathrm{DW}^{\varepsilon, \delta}\left(\mathbf{X}_{i}\right), \mathbf{X}_{i+1}-\mathbf{X}_{i}\right\rangle$ approximates $W^{\varepsilon, \delta}\left(\mathbf{X}_{\tau_{N}}\right)-$ $W^{\varepsilon, \delta}\left(\mathbf{X}_{0}\right)$ and provide an upper bound for the error term, similar to Lemma 2 in [4].

Lemma 3 Consider a two-node GI|GI|1 tandem queue satisfying Assumptions 1 and 2. Then, for any successful path $\mathbf{X}_{i}, i=0, \ldots, \tau_{N}$, it holds that

$$
\left|N \sum_{i=0}^{\tau_{N}-1}\left\langle D W^{\varepsilon, \delta}\left(\mathbf{X}_{i}\right), \mathbf{X}_{i+1}-\mathbf{X}_{i}\right\rangle-N\left(W^{\varepsilon, \delta}\left(\mathbf{X}_{\tau_{N}}\right)-W^{\varepsilon, \delta}\left(\mathbf{X}_{0}\right)\right)\right| \leq \frac{2 C_{1}^{2}}{\varepsilon N} \tau_{N},
$$

where $C_{1}=\sqrt{2}\left(\gamma+\theta^{*}\right)\left(\sqrt{2}+\sqrt{3} \max _{j}\left\{Q^{(j)}\right\}\right)<\infty$.

Proof We can bound $\left|\left\langle\mathrm{DW}^{\varepsilon, \delta}\left(\mathbf{X}_{i}\right), \mathbf{X}_{i+1}-\mathbf{X}_{i}\right\rangle-\left(W^{\varepsilon, \delta}\left(\mathbf{X}_{i+1}\right)-W^{\varepsilon, \delta}\left(\mathbf{X}_{i}\right)\right)\right|$ for each step $i$ by using the mean value theorem. Let $\mathbf{x}=\mathbf{X}_{i}$ and $\mathbf{y}=\mathbf{X}_{i+1}-\mathbf{X}_{i}$. By the mean value theorem, we have $W^{\varepsilon, \delta}(\mathbf{x}+\mathbf{y})-W^{\varepsilon, \delta}(\mathbf{x})=\left\langle\mathrm{DW}^{\varepsilon, \delta}(\mathbf{x}+\eta \mathbf{y}), \mathbf{y}\right\rangle$ for some $\eta \in[0,1]$. For convenience, we denote $R_{k}(\mathbf{x})=e^{-W_{k}^{\delta}(\mathbf{x}) / \varepsilon}$, so that $\rho_{k}(\mathbf{x})=$ $\frac{R_{k}(\mathbf{x})}{\sum_{j=1}^{3} R_{j}(\mathbf{x})}$, and $R_{k}(\mathbf{x}+\eta \mathbf{y})=R_{k}(\mathbf{x}) e^{-\left\langle\boldsymbol{\alpha}_{k}, \mathbf{y}\right\rangle \eta / \varepsilon}$, which implies that $\mathrm{DW}^{\varepsilon, \delta}(\mathbf{x}+\eta \mathbf{y})=$ $\sum_{k} \rho_{k}(\mathbf{x}+\eta \mathbf{y}) \boldsymbol{\alpha}_{k}=\sum_{k} \frac{R_{k}(\mathbf{x}+\eta \mathbf{y})}{\sum_{j=1}^{3} R_{j}(\mathbf{x}+\eta \mathbf{y})} \boldsymbol{\alpha}_{k}$. Thus, 


$$
\begin{aligned}
& \left|\left\langle\mathrm{DW}^{\varepsilon, \delta}(\mathbf{x}), \mathbf{y}\right\rangle-\left(W^{\varepsilon, \delta}(\mathbf{x}+\mathbf{y})-W^{\varepsilon, \delta}(\mathbf{x})\right)\right| \\
& =\left|\frac{\sum_{k=1}^{3} R_{k}(\mathbf{x})\left\langle\boldsymbol{\alpha}_{k}, \mathbf{y}\right\rangle}{\sum_{j=1}^{3} R_{j}(\mathbf{x})}-\frac{\sum_{k=1}^{3} R_{k}(\mathbf{x}+\eta \mathbf{y})\left\langle\boldsymbol{\alpha}_{k}, \mathbf{y}\right\rangle}{\sum_{j=1}^{3} R_{j}(\mathbf{x}+\eta \mathbf{y})}\right| \\
& =\left|\sum_{k=1}^{3} \frac{R_{k}(\mathbf{x})\left\langle\boldsymbol{\alpha}_{k}, \mathbf{y}\right\rangle}{\sum_{j=1}^{3} R_{j}(\mathbf{x})}\left(1-e^{-\left\langle\boldsymbol{\alpha}_{k}, \mathbf{y}\right\rangle \eta / \varepsilon} \frac{\sum_{j=1}^{3} R_{j}(\mathbf{x})}{\sum_{j=1}^{3} R_{j}(\mathbf{x}+\eta \mathbf{y})}\right)\right| \\
& \leq\left|\left\langle\mathrm{DW}^{\varepsilon, \delta}(\mathbf{x}), \mathbf{y}\right\rangle\right| \cdot \\
& \\
& \quad\left|\max \left\{1-e^{\left(\min _{k}\left\langle\boldsymbol{\alpha}_{k}, \mathbf{y}\right\rangle-\max _{k}\left\langle\boldsymbol{\alpha}_{k}, \mathbf{y}\right\rangle\right) \eta / \varepsilon}, e^{\left(\max _{k}\left\langle\boldsymbol{\alpha}_{k}, \mathbf{y}\right\rangle-\min _{k}\left\langle\boldsymbol{\alpha}_{k}, \mathbf{y}\right\rangle\right) \eta / \varepsilon}-1\right\}\right|,
\end{aligned}
$$

where the inequality follows by the definition of $\mathrm{DW}^{\varepsilon, \delta}(\mathbf{x})$, see (16), $\rho(\mathbf{x})$ and $R_{j}(\mathbf{x}+$ $\eta \mathbf{y}$ ), and by bounding $\left\langle\boldsymbol{\alpha}_{k}, \mathbf{y}\right\rangle$ by the minimum or maximum over $k$ (whichever is appropriate). We use the crude bounds

$$
\begin{aligned}
&\left|\mathrm{DW}^{\varepsilon, \delta}(\mathbf{x})\right| \leq \max _{k}\left|\boldsymbol{\alpha}_{k}\right| \leq \sqrt{2 \gamma^{2}+2\left(\theta^{*}\right)^{2}} \leq \sqrt{2}\left(\gamma+\theta^{*}\right), \\
&|\mathbf{y}| \leq \frac{1}{N} \max \left\{\sqrt{1+2\left(Q^{(0)}\right)^{2}+\left(\max \left\{Q^{(0)}, Q^{(1)}\right\}\right)^{2}},\right. \\
&\left.\sqrt{2+2\left(Q^{(1)}\right)^{2}+\left(\max \left\{Q^{(1)}, Q^{(2)}\right\}\right)^{2}}, \sqrt{1+3\left(Q^{(2)}\right)^{2}}\right\} \\
& \leq \frac{1}{N} \sqrt{2+3 \max _{j}\left\{\left(Q^{(j)}\right)^{2}\right\}} \leq \frac{\sqrt{2}+\sqrt{3} \max _{j}\left\{Q^{(j)}\right\}}{N},
\end{aligned}
$$

where the second inequality follows by considering all possible transitions. For example, in the case of an arrival the number of customers in the system changes by 1 , the residual inter-arrival time and the residual service time at queue 2 change by at most $Q^{(0)}$ and the residual service time at queue 1 changes by at $\operatorname{most} \max \left\{Q^{(0)}, Q^{(1)}\right\}$. Letting $C_{1}=\sqrt{2}\left(\gamma+\theta^{*}\right)\left(\sqrt{2}+\sqrt{3} \max _{j}\left\{Q^{(j)}\right\}\right)$, we find $\left|\left\langle\boldsymbol{\alpha}_{k}, \mathbf{y}\right\rangle\right| \leq C_{1}$ for all $k=1,2,3$, and so we can upper bound (24) by

$$
\begin{aligned}
\frac{C_{1}}{N}\left|\max \left\{1-e^{-2 \frac{C_{1} \eta}{\varepsilon N}}, e^{2 \frac{C_{1} \eta}{\varepsilon N}}-1\right\}\right| & =\frac{C_{1}}{N}\left(e^{2 \frac{C_{1} \eta}{\varepsilon N}}-1\right) \\
& =\frac{C_{1}}{N}\left(\frac{C_{1} \eta}{\varepsilon N}+\mathcal{O}\left(\frac{C_{1}^{2} \eta^{2}}{\varepsilon^{2} N^{2}}\right)\right) \leq \frac{2 C_{1}^{2}}{\varepsilon N^{2}},
\end{aligned}
$$

where the inequality holds for sufficiently large $\varepsilon N$, and hence we have found

$$
\left|N \sum_{i=0}^{\tau_{N}-1}\left\langle\mathrm{DW}^{\varepsilon, \delta}\left(\mathbf{X}_{i}\right), \mathbf{X}_{i+1}-\mathbf{X}_{i}\right\rangle-N\left(W^{\varepsilon, \delta}\left(\mathbf{X}_{\tau_{N}}\right)-W^{\varepsilon, \delta}\left(\mathbf{X}_{0}\right)\right)\right| \leq \frac{2 C_{1}^{2}}{\varepsilon N} \tau_{N} .
$$

Remark 15 The bound in Lemma 3 is very crude and better bounds can be obtained by considering all possible transitions separately. However, in order to show asymptotic efficiency the current bound is sufficient. 
Remark 16 Some solutions to $\mathbb{H}(\mathbf{x}, \boldsymbol{\alpha}) \geq 0$ in Table 1 use different values rather than $\theta^{*}$, for example, $\widetilde{\theta}^{(1)}$. It is easy to verify that for these different values, similar bounds as in Lemmas 2 and 3 can be obtained by using their definition. The only additional requirement is that the value replacing $\theta^{*}$ is finite. Note that $\theta^{*}$ is finite by Assumption 1.

In order to show asymptotic efficiency, we need an asymptotic result involving $\tau_{N}$, the total number of steps to reach level $N$ (given that level $N$ is reached before the system is empty). This is the subject of the following conjecture.

Conjecture 1 If $\sigma_{N}$ is a sequence of real numbers such that $\sigma_{N} \rightarrow 0$ when $N \rightarrow \infty$, then

$$
\limsup _{N \rightarrow \infty} \frac{1}{N} \log \mathbb{E}^{\mathbb{Q}}\left[e^{\tau_{N} \sigma_{N}} \mid \tau_{N}<\infty\right]=0 .
$$

Intuition We note that we can upper bound $\tau_{N}$ by $3 K_{N}$, where $K_{N}$ is the index of the first customer who reaches the overflow level $N$. Along the major part of the most likely path to reach the overflow level, the change of measure is very close to the state-independent change of measure as discussed in [2] (see also Examples 1 and 2). In Lemmas 3.2 and 4.1 of that same paper, it is shown that under this state-independent change of measure the system is unstable and $\frac{K_{N}}{N} \rightarrow$ $C<\infty$ with probability 1 . This means that the left-hand side of (25) would be upper bounded by $\lim \sup _{N \rightarrow \infty} \frac{1}{N} \log \mathbb{E}^{\mathbb{Q}}\left[e^{3 K_{N} \sigma_{N}} \mid \tau_{N}<\infty\right]$, which behaves as $\lim \sup _{N \rightarrow \infty} \frac{1}{N} \log \mathbb{E}^{\mathbb{Q}}\left[e^{3 C N \sigma_{N}}\right]=\lim _{N \rightarrow \infty} 3 C \sigma_{N}=0$. Also, suppose that (25) does not hold, i.e. $\lim \sup _{N \rightarrow \infty} \frac{1}{N} \log \mathbb{E}^{\mathbb{Q}}\left[e^{\tau_{N} \sigma_{N}} \mid \tau_{N}<\infty\right]>0$. This means that the random variable $\tau_{N}$, and also the random variable $K_{N}$, would grow much faster than $N$ as $N \rightarrow \infty$, which does not seem plausible.

For a mathematical proof of the conjecture, two problems remain. The first one is to show that the difference between the actual change of measure and the stateindependent change of measure (which is small along the most likely path) does not influence the validity of (25). The second problem is to show that for the stateindependent change of measure we indeed have $\lim _{\sup } \rightarrow \infty \frac{1}{N} \log \mathbb{E} \mathbb{Q}\left[e^{3 K_{N} \sigma_{N}} \mid \tau_{N}<\right.$ $\infty]=0$. Even if the change of measure were equal to the state-independent change of measure along the whole state space, we could not show this relation. Of course, there may be other possibilities to show that (25) holds.

We can now prove the main theorem of this section.

Theorem 2 Suppose we have a two-node GI|GI|1 tandem queue satisfying Assumptions 1 and 2. Then, under Conjecture 1 and Assumption 3, the change of measure based on $D W^{\varepsilon, \delta}(\mathbf{x})$ in Eq. (16) is asymptotically efficient.

Proof For a successful path $\mathcal{P}$, we have

$$
\log L(\mathcal{P})=N \sum_{i=0}^{\tau_{N}-1}\left\langle\mathrm{DW}^{\varepsilon, \delta}\left(\mathbf{X}_{i}\right), \mathbf{X}_{i+1}-\mathbf{X}_{i}\right\rangle-\sum_{i=0}^{\tau_{N}-1} \mathbb{H}\left(\mathbf{X}_{i}, \mathrm{DW}^{\varepsilon, \delta}\left(\mathbf{X}_{i}\right)\right)
$$




$$
\begin{aligned}
\leq & \frac{2 C_{1}^{2}}{\varepsilon N} \tau_{N}+N W^{\varepsilon, \delta}\left(\mathbf{X}_{\tau_{N}}\right)-N W^{\varepsilon, \delta}\left(\mathbf{X}_{0}\right) \\
& +\left(\theta^{*} \max \left\{Q^{(0)}, Q^{(1)}, Q^{(2)}\right\}+\gamma\right) e^{-\delta / \varepsilon} \tau_{N},
\end{aligned}
$$

where the second step follows from Lemmas 2 and 3. Define $\sigma_{N}=\frac{2 C_{1}^{2}}{\varepsilon N}+$ $\left(\theta^{*} \max \left\{Q^{(0)}, Q^{(1)}, Q^{(2)}\right\}+\gamma\right) e^{-\delta / \varepsilon}$. Then it follows that

$$
\log L(\mathcal{P}) \leq \tau_{N} \sigma_{N}+N W^{\varepsilon, \delta}\left(\mathbf{X}_{\tau_{N}}\right)-N W^{\varepsilon, \delta}\left(\mathbf{X}_{0}\right)
$$

Since $\mathbf{X}_{\tau_{N}} \in \delta_{e}$ for the successful path $\mathcal{P}$, we find, by using Lemma 1 ,

$$
\begin{aligned}
\log L(\mathcal{P}) & \leq \tau_{N} \sigma_{N}+N\left(\frac{\gamma}{N}-\delta\right)-N\left(\gamma\left(1-\frac{1}{N}\right)-3 \delta-\varepsilon \log (3)\right) \\
& =\tau_{N} \sigma_{N}+2 N \delta-\gamma(N-2)+\varepsilon N \log (3)
\end{aligned}
$$

and so we have

$$
\begin{aligned}
& \limsup _{N \rightarrow \infty} \frac{1}{N} \log \mathbb{E}^{\mathbb{Q}}\left[L(\mathcal{P})^{2} I(\mathcal{P})\right] \\
& \quad \leq \limsup _{N \rightarrow \infty} \frac{1}{N} \log \mathbb{E}^{\mathbb{Q}}\left[e^{2 \tau_{N} \sigma_{N}+4 N \delta-2 \gamma(N-2)+2 \varepsilon N \log (3)} I(\mathcal{P})\right] \\
& \quad=-2 \gamma+\limsup _{N \rightarrow \infty} \frac{1}{N} \log \left(\mathbb{E}^{\mathbb{Q}}\left[e^{2 \tau_{N} \sigma_{N}} \mid I(\mathcal{P})=1\right] \mathbb{P}^{\mathbb{Q}}(I(\mathcal{P})=1)\right) \\
& \leq-2 \gamma+\limsup _{N \rightarrow \infty} \frac{1}{N} \log \mathbb{E}^{\mathbb{Q}}\left[e^{2 \tau_{N} \sigma_{N}} \mid \tau_{N}<\infty\right],
\end{aligned}
$$

where the equality follows by Assumption 3 and the last step follows by noting that $\mathbb{P}^{\mathbb{Q}}(I(\mathcal{P})=1) \leq 1$. Using Conjecture 1 concludes the proof.

Remark 17 In the proof of Theorem 2, we bounded $N \sum_{i=0}^{\tau_{N}-1}\left\langle\mathrm{DW}^{\varepsilon, \delta}\left(\mathbf{X}_{i}\right), \mathbf{X}_{i+1}-\mathbf{X}_{i}\right\rangle$ and $\sum_{i=0}^{\tau_{N}-1} \mathbb{H}\left(\mathbf{X}_{i}, \mathrm{DW}^{\varepsilon, \delta}\left(\mathbf{X}_{i}\right)\right)$ separately, resulting in bounds involving the maximum support of the various distributions. Looking at Eqs. (18) and (19), we see, for example, that the new probability density functions themselves do not depend on $\mathcal{X}\left(\mathbf{X}_{i}\right) N$, and thus the likelihood ratio in (8) does not depend on $\mathcal{X}\left(\mathbf{X}_{i}\right) N$, even though $\mathcal{X}\left(\mathbf{X}_{i}\right)$ is needed in order to determine which probability density function is used. However, to use the mean value theorem in Lemma 3 we do need to keep this quantity throughout the proofs.

We can extend Theorem 2 to the following set of changes of measure; see also Remarks 11 and 16. We restrict ourselves to three regions only, since this is the easiest from an implementation perspective. Let

$$
\begin{aligned}
\operatorname{DW}^{\varepsilon, \delta}(\mathbf{x})= & \left(-\left(\rho_{1}(\mathbf{x})+\rho_{2}(\mathbf{x})\right) \gamma,-\rho_{1}(\mathbf{x}) \gamma,\left(\rho_{1}(\mathbf{x})+\rho_{2}(\mathbf{x})\right) \theta^{*},\right. \\
& \left.-\rho_{1}(\mathbf{x}) \bar{\alpha}_{1,1}-\rho_{2}(\mathbf{x}) \bar{\alpha}_{1,2},-\rho_{1}(\mathbf{x}) \bar{\alpha}_{2,1}-\rho_{2}(\mathbf{x}) \bar{\alpha}_{2,2}\right),
\end{aligned}
$$


where

$$
\begin{aligned}
& \bar{\alpha}_{1,1} \in\left[0,-\bar{\alpha}_{2,1}-\theta^{*}\right], \\
& \bar{\alpha}_{2,1} \in\left[-\widetilde{\theta}^{(2)},-\theta^{*}\right], \\
& \bar{\alpha}_{1,2} \in\left[-\widetilde{\theta}^{(1)},-\theta^{*}\right], \\
& \bar{\alpha}_{2,2} \in\left[0,-\bar{\alpha}_{1,2}-\theta^{*}\right] .
\end{aligned}
$$

Note that when queue $j$ is the bottleneck queue, $\widetilde{\theta}^{(j)}=\theta^{*}$ and hence along the most likely path still the state-independent change of measure studied in $[2,9,11]$ is used.

We leave the proof of the following theorem to the reader, as this only requires some small modifications in the proofs of Lemmas 2, 3 and Theorem 2.

Theorem 3 Suppose we have a two-node GI|GI|1 tandem queue satisfying Assumptions 1 and 2. Then, under Conjecture 1 and Assumption 3, the change of measure based on $D W^{\varepsilon, \delta}(\mathbf{x})$ in Eq. (26) is asymptotically efficient.

\subsection{The $d$-node $G|| G|| 1$ tandem queue}

In this section, we present the steps to follow in order to find a state-dependent change of measure for the $d$-node $G I|G I| 1$ tandem queue. As we needed a conjecture for the case $d=2$, we also need a conjecture for the more general case. We will formulate similar lemmas as in Sect. 3.2 but omit the proofs, as these are just extensions of the proofs for the two-node tandem queue and therefore do not require any additional techniques.

\subsubsection{Solutions to $\mathbb{H}(\mathrm{x}, \alpha) \geq 0$ for all possible $\mathrm{x}$}

For the $d$-node tandem queue we have, for $\mathbf{x} \neq \mathbf{X}_{0}$,

$$
\begin{aligned}
& \mathbb{H}(\mathbf{x}, \boldsymbol{\alpha})=-\mathcal{X}(\mathbf{x}) N\left(\bar{\alpha}_{0}+\sum_{k=1}^{d} \bar{\alpha}_{k} \mathbb{1}\left\{x_{k}>0\right\}\right) \\
& \quad+\left\{\begin{array}{cl}
\alpha_{1}-\Lambda_{A}\left(-\bar{\alpha}_{0}\right)-\Lambda_{B^{(1)}}\left(-\bar{\alpha}_{1} \mathbb{1}\left\{x_{1}=0\right\}\right) & \text { if } \mathcal{X}(\mathbf{x})=\bar{x}_{0}, \\
\alpha_{j+1}-\alpha_{j}-\Lambda_{B^{(j)}}\left(-\bar{\alpha}_{j} \mathbb{1}\left\{x_{j}>\frac{1}{N}\right\}\right) & \text { if } \mathcal{X}(\mathbf{x})=\bar{x}_{j}, j=1, \ldots, d-1, \\
-\Lambda_{B^{(j+1)}}\left(-\bar{\alpha}_{j+1} \mathbb{1}\left\{x_{j+1}=0\right\}\right) & \text { if } \mathcal{X}(\mathbf{x})=\bar{x}_{d}, \\
-\alpha_{d}-\Lambda_{B^{(d)}}\left(-\bar{\alpha}_{d} \mathbb{1}\left\{x_{d}>\frac{1}{N}\right\}\right) &
\end{array}\right.
\end{aligned}
$$

and for $\mathbf{x}=\mathbf{X}_{0}$

$$
\mathbb{H}\left(\mathbf{X}_{0}, \boldsymbol{\alpha}\right)=-\Lambda_{A}\left(-\bar{\alpha}_{0}\right)-\Lambda_{B^{(1)}}\left(-\bar{\alpha}_{1}\right)
$$

Some possible solutions to $\mathbb{H}(\mathbf{x}, \boldsymbol{\alpha}) \geq 0$, similar to the solutions presented in Table 2 , can be found in Table 3 (including the trivial solution $\boldsymbol{\alpha}=\mathbf{0}$ ). Similarly to Table 2, the solutions presented consider cases where some $x_{j}>0$. Due to the construction that 
Table 3 Some solutions to $\mathbb{H}(\mathbf{x}, \boldsymbol{\alpha}) \geq 0$ for all $\mathbf{x}$

\begin{tabular}{lll}
\hline Region of validity & Nonzero $\alpha_{i}$ 's & Nonzero $\bar{\alpha}_{i}$ 's \\
\hline$x_{1}>0$ & $\alpha_{1}=\Lambda_{A}\left(-\theta^{*}\right)$ & $\bar{\alpha}_{0}=-\bar{\alpha}_{1}=\theta^{*}$ \\
$\vdots$ & $\vdots$ & $\vdots$ \\
$x_{j}>0$ & $\alpha_{1}=\cdots=\alpha_{j}=\Lambda_{A}\left(-\theta^{*}\right)$ & $\bar{\alpha}_{0}=-\bar{\alpha}_{j}=\theta^{*}$ \\
$\vdots$ & $\vdots$ & $\vdots$ \\
$x_{d}>0$ & $\alpha_{1}=\cdots=\alpha_{d}=\Lambda_{A}\left(-\theta^{*}\right)$ & $\bar{\alpha}_{0}=-\bar{\alpha}_{d}=\theta^{*}$ \\
All $\mathbf{x}$ & &
\end{tabular}

All $\alpha_{i}$ 's and $\bar{\alpha}_{i}$ 's that are not explicitly mentioned in this table are equal to 0 in that particular case

is used, using the minimum of all subsolutions, these solutions also cover the interior of the state space.

Clearly, solutions similar to the other solutions presented in Table 1 also exist. For example, define $\widetilde{\theta}^{(1)}=\sup \left\{\theta: \Lambda_{A}\left(-\theta^{*}\right)+\Lambda_{B^{(1)}}(\theta) \leq 0\right\}$. When $x_{1}>0$, another possibility would be $\bar{\alpha}_{1} \in\left[-\widetilde{\theta}^{(1)},-\theta^{*}\right]$ and $\bar{\alpha}_{j} \in\left[0,-\sum_{i \neq j} \bar{\alpha}_{i}\right], j>1$. Thus, when $x_{j}>0$, we define $\widetilde{\theta}^{(j)}=\sup \left\{\theta: \Lambda_{A}\left(-\theta^{*}\right)+\Lambda_{B^{(j)}}(\theta) \leq 0\right\}$ and another possibility would be $\bar{\alpha}_{j} \in\left[-\widetilde{\theta}^{(j)},-\theta^{*}\right]$ and, for $k>0, \bar{\alpha}_{k} \in\left[0,-\sum_{i \neq k} \bar{\alpha}_{i}\right], k \neq j$.

\subsubsection{Construction of $W(\mathrm{x})$}

As for the two-node tandem queue, there is no solution for $\mathbb{H}(\mathbf{x}, \boldsymbol{\alpha}) \geq 0$ that holds for all $\mathbf{x}$. Therefore, a different change of measure needs to be applied in different 'regions'. In this case, the following vectors are used to specify the different changes of measure in each of the 'regions':

$$
\begin{aligned}
\boldsymbol{\alpha}_{1} & =-\gamma\left(\mathbf{e}_{1}+\cdots+\mathbf{e}_{d}\right)+\theta^{*}\left(\overline{\mathbf{e}}_{0}-\overline{\mathbf{e}}_{d}\right) \\
& \vdots \\
\boldsymbol{\alpha}_{j} & =-\gamma\left(\mathbf{e}_{1}+\cdots+\mathbf{e}_{d-(j-1)}\right)+\theta^{*}\left(\overline{\mathbf{e}}_{0}-\overline{\mathbf{e}}_{d-(j-1)}\right), \\
& \vdots \\
\boldsymbol{\alpha}_{d} & =-\gamma \mathbf{e}_{1}+\theta^{*}\left(\overline{\mathbf{e}}_{0}-\overline{\mathbf{e}}_{1}\right) \\
\boldsymbol{\alpha}_{d+1} & =(0, \ldots, 0)
\end{aligned}
$$

From this, we find $d+1$ functions $W_{k}^{\delta}(\mathbf{x})=\left\langle\boldsymbol{\alpha}_{k}, \mathbf{x}\right\rangle+\gamma-k \delta$, for $k=1, \ldots, d+1$, for $\delta>0$, and their minimum

$$
W^{\delta}(\mathbf{x})=W_{1}^{\delta}(\mathbf{x}) \wedge \cdots \wedge W_{d+1}^{\delta}(\mathbf{x})
$$

which again is a piecewise affine function that equals $W_{k}^{\delta}(\mathbf{x})$ in 'region' $k$. The constant $\gamma$ in $W_{k}^{\delta}(\mathbf{x})$ is included to satisfy the properties of the subsolution, and the 
subtraction of $k \delta$ is needed so that the minimum function $W^{\delta}(\mathbf{x})$ is uniquely attained at each of the boundaries.

To make the minimum function a continuously differentiable function, we apply a similar mollification procedure as for the two-node $G I|G I| 1$ tandem queue. This mollification 'removes' the regions and ensures that a derivative exists throughout the whole parameter space. We let

$$
W^{\varepsilon, \delta}(\mathbf{x})=-\varepsilon \log \sum_{k=1}^{d+1} e^{-W_{k}^{\delta}(\mathbf{x}) / \varepsilon}
$$

When $\varepsilon \rightarrow 0, W^{\varepsilon, \delta}(\mathbf{x}) \rightarrow W_{1}^{\delta}(\mathbf{x}) \wedge \cdots \wedge W_{d+1}^{\delta}(\mathbf{x})$. The assumptions on $\varepsilon$ and $\delta$ can be found in Assumption 3. Similarly to Lemma 1, we see that $W^{\varepsilon, \delta}(\mathbf{x})$ satisfies properties 3 and 4 in and below Definition 2 .

Lemma 4 Under Assumption 1, we have, for the d-node GI $|G I| 1$ tandem queue, that $W^{\varepsilon, \delta}(\mathbf{x}) \leq \frac{\gamma}{N}-\delta$ for $\mathbf{x} \in \delta_{e}$ and $\gamma\left(1-\frac{1}{N}\right)-(d+1) \delta-\varepsilon \log (d+1) \leq W^{\varepsilon, \delta}\left(\mathbf{X}_{0}\right) \leq$ $\gamma-(d+1) \delta$.

The change of measure will be based on the gradient of $W^{\varepsilon, \delta}(\mathbf{x})$. From (28), it follows that

$$
\mathrm{DW}^{\varepsilon, \delta}(\mathbf{x})=\sum_{k=1}^{d+1} \rho_{k}(\mathbf{x}) \boldsymbol{\alpha}_{k}
$$

where $\rho_{k}(\mathbf{x})$ is defined similarly to in (17).

\subsubsection{The change of measure}

As for the 2-node tandem queue, we show that the change of measure based on $W_{k}^{\delta}(\mathbf{x})$ results in the state-independent change of measure studied in $[2,9,11]$ in some particular cases. This gives some insight into the change of measure that is applied in the mollified function $W^{\varepsilon, \delta}(\mathbf{x})$.

Choosing $\operatorname{DW}(\mathbf{x})$ in (7) as a constant vector $\boldsymbol{\alpha}=\left(\alpha_{1}, \ldots, \alpha_{d}, \bar{\alpha}_{0}, \ldots, \bar{\alpha}_{d}\right)$, the change of measure can be written in terms of $\bar{\alpha}_{0}, \ldots, \bar{\alpha}_{d}$ as

$$
\begin{aligned}
& \mathrm{d} \bar{F}_{V_{X}(\mathbf{x})}(\mathbf{v} \mid \mathbf{x})
\end{aligned}
$$

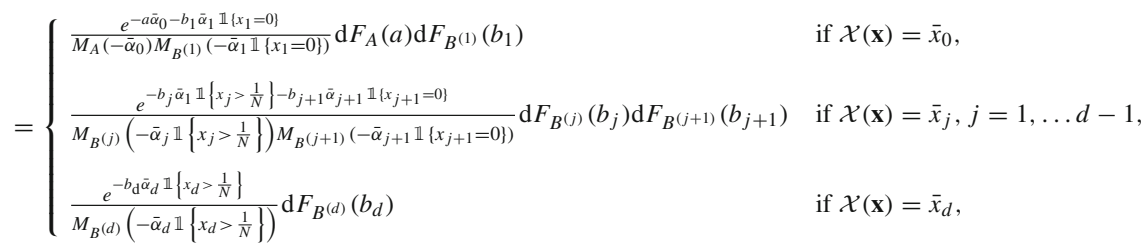


for $\mathbf{x} \neq \mathbf{X}_{0}$ and

$$
\mathrm{d} \bar{F}_{V_{X}\left(\mathbf{X}_{0}\right)}\left(\mathbf{v} \mid \mathbf{X}_{0}\right)=\frac{e^{-a \bar{\alpha}_{0}-b_{1} \bar{\alpha}_{1}}}{M_{A}\left(-\bar{\alpha}_{0}\right) M_{B^{(1)}}\left(-\bar{\alpha}_{1}\right)} \mathrm{d} F_{A}(a) \mathrm{d} F_{B^{(1)}}\left(b_{1}\right)
$$

We now consider the cases where our change of measure based on $W^{\varepsilon, \delta}(\mathbf{x})$ gets close, as $N \rightarrow \infty$, to an 'affine' change of measure as above, that is, based on some $W_{k}^{\delta}(\mathbf{x})$ with constant gradient. Then, if we let $\alpha=W_{d-(j-1)}^{\delta}(\mathbf{x})=\boldsymbol{\alpha}_{d-(j-1)}$, see (27), it follows that indeed the resulting change of measure equals the state-independent change of measure studied in $[2,9,11]$ if queue $j$ is the bottleneck queue.

\subsubsection{Asymptotic efficiency}

To show that asymptotic efficiency also holds for the $d$-node $G I|G I| 1$ tandem queue, assuming that Conjecture 1 is correct, we need the following two lemmas.

Lemma 5 Under Assumptions 1 and 2, we have, for the d-node GI|GI|1 tandem queue, for all possible $\mathbf{x}$,

$$
\mathbb{H}\left(\mathbf{x}, D W^{\varepsilon, \delta}(\mathbf{x})\right) \geq-\left(\theta^{*} \max \left\{Q^{(0)}, \ldots, Q^{(d)}\right\}+\gamma\right) e^{-\delta / \varepsilon} .
$$

Lemma 6 Suppose we have ad-node $G I|G I| 1$ tandem queue satisfying Assumptions 1 and 2. Then, for any successful path $\mathbf{X}_{i}, i=0, \ldots, \tau_{N}$, it holds for all $\mathbf{X}_{\tau_{N}}$ that

$$
\left|N \sum_{i=0}^{\tau_{N}-1}\left\langle D W^{\varepsilon, \delta}\left(\mathbf{X}_{i}\right), \mathbf{X}_{i+1}-\mathbf{X}_{i}\right\rangle-N\left(W^{\varepsilon, \delta}\left(\mathbf{X}_{\tau_{N}}\right)-W^{\varepsilon, \delta}\left(\mathbf{X}_{0}\right)\right)\right| \leq \frac{2 C_{2}^{2}}{\varepsilon N} \tau_{N},
$$

where $C_{2}=\sqrt{2}\left(\gamma+\theta^{*}\right)\left(\sqrt{2}+\sqrt{3} \max _{j}\left\{Q^{(j)}\right\}\right)<\infty$.

Using Lemmas 4-6, we can prove the following result.

Theorem 4 Suppose we have a d-node GI $\mid$ GI $\mid 1$ tandem queue satisfying Assumptions 1 and 2. Then, under Conjecture 1 and Assumption 3, the change of measure based on $D W^{\varepsilon, \delta}(\mathbf{x})$ in Eq. (29) is asymptotically efficient.

Remark 18 We could also extend Theorem 4, as we did for the two-node tandem queue in Theorem 3, and we claim that asymptotic efficiency also holds in that case under the same conditions as in Theorem 4.

\section{Numerical results for the two-node tandem queue}

In this section, we present numerical results for the two-node tandem queue to illustrate that the proposed method indeed works. In each of the examples below, we use (16) 
for the change of measure. We recall that the estimator for $p_{N}$ is $L(\mathcal{P}) I(\mathcal{P})$ and hence we will define the following estimator, which is obtained via simulation,

$$
\hat{p}_{N}=\frac{1}{S} \sum_{i=1}^{S} L(i) I(i)
$$

where $S$ is the total number of simulation runs, $L(i)$ is the likelihood ratio in simulation $i$ and $I(i)$ indicates whether level $N$ has been reached in simulation $i$ or not.

In all tables given below, RE is the relative error, i.e., the standard deviation of the estimator divided by its mean,

$$
\mathrm{RE}=\frac{\sqrt{\frac{1}{S-1} \sum_{i=1}^{S}\left(L(i) I(i)-\hat{p}_{N}\right)^{2}}}{\sqrt{S} \hat{p}_{N}},
$$

and AE denotes

$$
\mathrm{AE}=\frac{\log \frac{1}{S} \sum_{i=1}^{S} L^{2}(i) I(i)}{\log \frac{1}{S} \sum_{i=1}^{S} L(i) I(i)}
$$

Thus, if the change of measure is asymptotically efficient, AE should converge to 2 when $N$ tends to infinity; see Definition 3. Furthermore, in our tables we will include the number of times the overflow level has been reached (out of a total of $S=10^{6}$ simulation runs) and the (rounded) simulation time in seconds. Note that the latter quantity is only there for reference and does not indicate if the estimator is asymptotically efficient or not.

To use the change of measure based on (16), we need to choose $\varepsilon$ and $\delta$ so that they satisfy Assumption 3. It is not trivial to find suitable $\varepsilon$ and $\delta$ that satisfy all requirements; we only explored several possibilities, while many more exist. In all the examples below, we set $\varepsilon$ proportional to $\frac{1}{\sqrt{N}}$ and $\delta=-\varepsilon \log \varepsilon$, unless the condition in Remark 19 is not satisfied.

Remark 19 For the choice $\delta=-\varepsilon \log \varepsilon$, it may happen for small values of $N$ that $W^{\delta}(\mathbf{x})$ does not attain $W_{k}^{\delta}(\mathbf{x})$ for some $k$ in the 'region' where it is supposed to be attained. For example, consider $W_{2}^{\delta}(\mathbf{x})<W_{1}^{\delta}(\mathbf{x})$, which is equivalent to

$$
x_{2}<\frac{\theta^{*}\left(\bar{x}_{1}-\bar{x}_{2}\right)}{\gamma}+\frac{\delta}{\gamma} \text {. }
$$

Taking into account that $W_{2}^{\delta}(\mathbf{x})$ is designed for the case $x_{2}=0$, the right-hand side of this equation should be positive. Thus, we need $\delta>\frac{Q^{(2)}}{N} \theta^{*}$ (recall that $Q^{(2)}$ is an upper bound on the support of the service times at queue 2 ). When we also consider $W_{1}^{\delta}(\mathbf{x})<W_{3}^{\delta}(\mathbf{x})$ and $W_{2}^{\delta}(\mathbf{x})<W_{3}^{\delta}(\mathbf{x})$, it turns out that we actually need

$$
\delta>\frac{\max \left\{Q^{(1)}, Q^{(2)}\right\} \theta^{*}}{N} .
$$


Table $4 D|U| 1-\cdot|U| 1$ tandem queue when both queues are the bottleneck queue. We choose $A=1.1$, $B^{(1)} \sim U[0,2], B^{(2)} \sim U[0,2]$ and $\varepsilon=\frac{0.06}{\sqrt{N}}$. We find $\theta^{*}=\theta_{1}=\theta_{2}=0.6073$. The number of simulations is $10^{6}$

\begin{tabular}{llllcc}
\hline$N$ & $\hat{p}_{N}$ & RE & AE & \#Overflow & Time (s) \\
\hline 20 & $1.3448 \mathrm{e}-03$ & 0.0877 & 0.6465 & 680910 & 93 \\
60 & $1.7785 \mathrm{e}-14$ & 0.0536 & 1.7485 & 580520 & 307 \\
100 & $8.7767 \mathrm{e}-26$ & 0.0290 & 1.8833 & 455950 & 451 \\
140 & $3.2087 \mathrm{e}-37$ & 0.0112 & 1.9424 & 339723 & 520 \\
180 & $1.0567 \mathrm{e}-48$ & 0.0048 & 1.9711 & 242793 & 533 \\
220 & $3.1997 \mathrm{e}-60$ & 0.0037 & 1.9803 & 169426 & 512 \\
260 & $9.4889 \mathrm{e}-72$ & 0.0038 & 1.9833 & 118241 & 478 \\
300 & $2.7188 \mathrm{e}-83$ & 0.0044 & 1.9843 & 81788 & 440 \\
340 & $7.6193 \mathrm{e}-95$ & 0.0053 & 1.9844 & 57009 & 405 \\
380 & $2.1168 \mathrm{e}-106$ & 0.0067 & 1.9843 & 40519 & 376 \\
420 & $5.9068 \mathrm{e}-118$ & 0.0084 & 1.9842 & 29311 & 354 \\
460 & $1.5638 \mathrm{e}-129$ & 0.0110 & 1.9838 & 20811 & 333 \\
500 & $4.2998 \mathrm{e}-141$ & 0.0140 & 1.9836 & 15300 & 317 \\
\hline
\end{tabular}

Therefore, when $\delta=-\varepsilon \log \varepsilon$ does not satisfy (32), in our numerical experiments, we actually set $\delta=\frac{\max \left\{Q^{(1)}, Q^{(2)}\right\} \theta^{*}}{N^{0.95}}$. We choose the denominator to be $N^{0.95}$, but of course other choices are possible to obtain the strict inequality in (32). Unless mentioned otherwise, only for $N=20$ this different value for $\delta$ is used. (Note that for a $d$-node tandem queue, a similar condition exists; $\delta>\frac{\max \left\{Q^{(1)}, \ldots, Q^{(d)}\right\} \theta^{*}}{N}$.)

We consider two types of tandem queues, a $D|U| 1-\cdot|U| 1$ tandem queue and a $M|U| 1-\cdot|U| 1$ tandem queue, and in both cases we vary the bottleneck queue. Starting with a $D|U| 1-\cdot|U| 1$ tandem queue, we first consider the case where the $\theta$-bottleneck queue is not unique, i.e., the service times at both queues have the same distribution with the same parameters. In our case, both service times have a uniform distribution on the interval [0,2]. Similar cases are known to fail when using a state-independent change of measure, see [2,3], and thus this is an interesting case to consider. The results can be found in Table 4. In this table, we see results that clearly support the theoretical results of the estimator being asymptotically efficient; at first the relative error is decreasing, after which it slowly increases. We also see that the number of times the overflow level $N$ is reached is decreasing with $N$. This behaviour may seem strange, but it can be seen in all of the results in this section. Given our assumptions on $\varepsilon$ and $\delta$, we note that $\delta N \rightarrow \infty$ and hence 'region' 3-where no change of measure is applied—is increasing in size. Therefore, we can expect that as $N$ increases it becomes increasingly more difficult to reach the overflow level, even though we have asymptotic efficiency. In particular, we observed that when a system has small server utilizations, it may be hard to escape the 'region' of the state space where (almost) no change of measure is applied.

Next, we let one of the queues be the bottleneck queue. In Tables 5 and 6, queue 1 and queue 2 are the bottleneck queue, respectively. Here, we changed one of the service 
Table $5 D|U| 1-\cdot|U| 1$ tandem queue when queue 1 is the bottleneck queue. We choose $A=1.1, B^{(1)} \sim$ $U[0,2], B^{(2)} \sim U[0.5,1.5]$ and $\varepsilon=\frac{0.07}{\sqrt{N}}$. We find $\theta^{*}=\theta_{1}=0.6073, \theta_{2}=2.5215$. The number of simulations is $10^{6}$

\begin{tabular}{lllllr}
\hline$N$ & $\hat{p}_{N}$ & RE & AE & \#Overflow & Time (s) \\
\hline 20 & $7.1456 \mathrm{e}-04$ & 0.4771 & 0.2971 & 854566 & 838 \\
60 & $2.2719 \mathrm{e}-15$ & 0.0852 & 1.7363 & 802889 & 1761 \\
100 & $1.0067 \mathrm{e}-26$ & 0.0977 & 1.8469 & 764219 & 1781 \\
140 & $3.4432 \mathrm{e}-38$ & 0.0811 & 1.8981 & 708956 & 1952 \\
180 & $9.0425 \mathrm{e}-50$ & 0.0322 & 1.9385 & 632963 & 2235 \\
220 & $2.2366 \mathrm{e}-61$ & 0.0088 & 1.9688 & 540583 & 2566 \\
260 & $5.6593 \mathrm{e}-73$ & 0.0111 & 1.9711 & 441182 & 2838 \\
300 & $1.3905 \mathrm{e}-84$ & 0.0043 & 1.9847 & 346529 & 3059 \\
340 & $3.4348 \mathrm{e}-96$ & 0.0046 & 1.9859 & 262769 & 3219 \\
380 & $8.6114 \mathrm{e}-108$ & 0.0052 & 1.9865 & 195534 & 3316 \\
420 & $2.1157 \mathrm{e}-119$ & 0.0056 & 1.9873 & 142657 & 3382 \\
460 & $5.3385 \mathrm{e}-131$ & 0.0128 & 1.9830 & 103512 & 3408 \\
500 & $1.3122 \mathrm{e}-142$ & 0.0086 & 1.9868 & 75186 & 3374 \\
\hline
\end{tabular}

Table $6 D|U| 1-\cdot|U| 1$ tandem queue when queue 2 is the bottleneck queue. We choose $A=1.1, B^{(1)} \sim$ $U[0.5,1.5], B^{(2)} \sim U[0,2]$ and $\varepsilon=\frac{0.01}{\sqrt{N}}$. We find $\theta^{*}=\theta_{2}=0.6073, \theta_{1}=2.5215$. The number of simulations is $10^{6}$

\begin{tabular}{lllllr}
\hline$N$ & $\hat{p}_{N}$ & RE & AE & \#Overflow & Time (s) \\
\hline 20 & $7.8819 \mathrm{e}-05$ & 0.0125 & 1.4655 & 478564 & 87 \\
60 & $2.0245 \mathrm{e}-16$ & 0.0138 & 1.8546 & 443297 & 245 \\
100 & $5.0153 \mathrm{e}-28$ & 0.0102 & 1.9250 & 426668 & 390 \\
140 & $1.2576 \mathrm{e}-39$ & 0.0117 & 1.9449 & 416708 & 550 \\
180 & $3.1214 \mathrm{e}-51$ & 0.0106 & 1.9593 & 408285 & 679 \\
220 & $7.8784 \mathrm{e}-63$ & 0.0129 & 1.9642 & 403760 & 808 \\
260 & $1.9570 \mathrm{e}-74$ & 0.0092 & 1.9738 & 398371 & 931 \\
300 & $4.7882 \mathrm{e}-86$ & 0.0237 & 1.9678 & 548146 & 1433 \\
340 & $1.2159 \mathrm{e}-97$ & 0.0227 & 1.9720 & 505585 & 1495 \\
380 & $2.9018 \mathrm{e}-109$ & 0.0074 & 1.9840 & 462204 & 1526 \\
420 & $7.4330 \mathrm{e}-121$ & 0.0107 & 1.9828 & 422362 & 1541 \\
460 & $1.8204 \mathrm{e}-132$ & 0.0061 & 1.9880 & 385678 & 1540 \\
500 & $4.6143 \mathrm{e}-144$ & 0.0107 & 1.9856 & 350976 & 1525 \\
\hline
\end{tabular}

time distributions of the previous example from $U[0,2]$ to $U[0.5,1.5]$ so that there is a unique $\theta$-bottleneck queue, but the server utilizations of both queues remain the same. When queue 1 is the bottleneck queue, we see that the relative error is still very large for $N=20$, but for larger values of $N$ the theoretical results are supported. Note that in this case $\delta=-\varepsilon \log \varepsilon$ for all values of $N$. We also see from this table that the 
simulation time is increasing, even though the number of times the overflow level is reached is decreasing. This is due to the fact that we have to reach a higher overflow level $N$. When queue 2 is the bottleneck queue, we have considered a much smaller value for $\varepsilon$, and thus $\delta$ differs from being $-\varepsilon \log \varepsilon$ for $N=20, \ldots, 260$. This can also be seen from the table, by noting that both the number of times the overflow is reached and the simulation time increase at $N=300$. Besides this difference, the results in the table clearly support the theoretical results.

Lastly, we consider a $M|U| 1-\cdot|U| 1$ tandem queue in Tables 7, 8 and 9. We emphasize that the inter-arrival times are exponentially distributed, which means an unbounded support, and, hence, this is not covered by our theoretical results. Again, we start with the case where there is no unique bottleneck queue in Table 7 . We see from this table that the relative error remains roughly constant, suggesting asymptotic efficiency. In Table 8, when queue 1 is the bottleneck queue, and in Table 9, when queue 2 is the bottleneck queue, we see that the relative error is slightly increasing, but still suggesting asymptotic efficiency. We remark that in Table $9 \delta$ does not equal $-\varepsilon \log \varepsilon$ for $N=20, \ldots, 100$, which can be seen by an increasing number of times level $N$ is reached for $N=140$.

Even though we do not have a proof of asymptotic efficiency for unbounded supports, which is the case for the inter-arrival times in Tables 7, 8 and 9, all these tables show good results that suggest asymptotic efficiency of the estimator. We also did some experiments with exponentially distributed service times, where, regardless of (32) in Remark 19, we set $\delta=-\varepsilon \log \varepsilon$, but we did not obtain good results there. This is most likely due to the fact that for Markovian service times (and unbounded service times in general), the condition on $\delta$, see (32), cannot hold. As a result, it could happen that, for example, $W_{2}^{\delta}(\mathbf{x})>W_{1}^{\delta}(\mathbf{x})$ when $x_{2}=0$, see also Remark 19, leading for $x_{2}=0$

Table $7 M|U| 1-\cdot|U| 1$ tandem queue when both queues are the bottleneck queue. We choose $A \sim \exp (0.5)$, $B^{(1)} \sim U[0,3], B^{(2)} \sim U[0,3]$ and $\varepsilon=\frac{0.025}{\sqrt{N}}$. We find $\theta^{*}=\theta_{1}=\theta_{2}=0.2690$. The number of simulations is $10^{6}$

\begin{tabular}{llllll}
\hline$N$ & $\hat{p}_{N}$ & RE & AE & \#Overflow & Time (s) \\
\hline 20 & $1.9166 \mathrm{e}-03$ & 0.0065 & 1.3957 & 362952 & 16 \\
60 & $1.8257 \mathrm{e}-10$ & 0.0087 & 1.8067 & 322186 & 53 \\
100 & $1.0024 \mathrm{e}-17$ & 0.0088 & 1.8885 & 231546 & 68 \\
140 & $4.5750 \mathrm{e}-25$ & 0.0082 & 1.9247 & 175071 & 74 \\
180 & $1.9576 \mathrm{e}-32$ & 0.0096 & 1.9379 & 137692 & 76 \\
220 & $7.9868 \mathrm{e}-40$ & 0.0080 & 1.9537 & 109868 & 76 \\
260 & $3.1289 \mathrm{e}-47$ & 0.0080 & 1.9611 & 89605 & 74 \\
300 & $1.2203 \mathrm{e}-54$ & 0.0106 & 1.9620 & 74172 & 71 \\
340 & $4.5110 \mathrm{e}-62$ & 0.0080 & 1.9704 & 61372 & 68 \\
380 & $1.6843 \mathrm{e}-69$ & 0.0083 & 1.9731 & 51929 & 65 \\
420 & $6.0889 \mathrm{e}-77$ & 0.0081 & 1.9761 & 43639 & 62 \\
460 & $2.2324 \mathrm{e}-84$ & 0.0083 & 1.9779 & 37347 & 59 \\
500 & $8.1358 \mathrm{e}-92$ & 0.0087 & 1.9793 & 32318 & 56 \\
\hline
\end{tabular}


Table $8 M|U| 1-\cdot|U| 1$ tandem queue when queue 1 is the bottleneck queue. We choose $A \sim \exp (0.5)$, $B^{(1)} \sim U[0,3], B^{(2)} \sim U[0,2]$ and $\varepsilon=\frac{0.025}{\sqrt{N}}$. We find $\theta^{*}=\theta_{1}=0.2690, \theta_{2}=0.8966$. The number of simulations is $10^{6}$

\begin{tabular}{llllll}
\hline$N$ & $\hat{p}_{N}$ & RE & AE & \#Overflow & Time (s) \\
\hline 20 & $3.3042 \mathrm{e}-04$ & 0.0024 & 1.7592 & 249800 & 13 \\
60 & $1.1016 \mathrm{e}-11$ & 0.0027 & 1.9160 & 217878 & 39 \\
100 & $3.6616 \mathrm{e}-19$ & 0.0032 & 1.9435 & 150647 & 46 \\
140 & $1.2177 \mathrm{e}-26$ & 0.0037 & 1.9550 & 111215 & 48 \\
180 & $4.0285 \mathrm{e}-34$ & 0.0041 & 1.9628 & 84826 & 48 \\
220 & $1.3518 \mathrm{e}-41$ & 0.0046 & 1.9671 & 67284 & 47 \\
260 & $4.5013 \mathrm{e}-49$ & 0.0050 & 1.9706 & 54098 & 45 \\
300 & $1.4843 \mathrm{e}-56$ & 0.0055 & 1.9731 & 43870 & 43 \\
340 & $4.9227 \mathrm{e}-64$ & 0.0060 & 1.9751 & 36140 & 40 \\
380 & $1.6372 \mathrm{e}-71$ & 0.0069 & 1.9762 & 30178 & 38 \\
420 & $5.4779 \mathrm{e}-79$ & 0.0072 & 1.9780 & 25657 & 36 \\
460 & $1.8101 \mathrm{e}-86$ & 0.0077 & 1.9793 & 21731 & 34 \\
500 & $6.0619 \mathrm{e}-94$ & 0.0084 & 1.9802 & 18566 & 32 \\
\hline
\end{tabular}

Table $9 M|U| 1-\cdot|U| 1$ tandem queue when queue 2 is the bottleneck queue. We choose $A \sim \exp (0.5)$, $B^{(1)} \sim U[0,2], B^{(2)} \sim U[0,3]$ and $\varepsilon=\frac{0.01}{\sqrt{N}}$. We find $\theta^{*}=\theta_{2}=0.2690, \theta_{1}=0.8966$. The number of simulations is $10^{6}$

\begin{tabular}{lllllr}
\hline$N$ & $\hat{p}_{N}$ & RE & AE & \#Overflow & Time (s) \\
\hline 20 & $3.2750 \mathrm{e}-04$ & 0.0038 & 1.6617 & 157082 & 9 \\
60 & $1.0824 \mathrm{e}-11$ & 0.0039 & 1.8891 & 144253 & 27 \\
100 & $3.6318 \mathrm{e}-19$ & 0.0039 & 1.9349 & 140723 & 43 \\
140 & $1.2032 \mathrm{e}-26$ & 0.0026 & 1.9656 & 199660 & 84 \\
180 & $4.0069 \mathrm{e}-34$ & 0.0032 & 1.9688 & 159602 & 88 \\
220 & $1.3319 \mathrm{e}-41$ & 0.0036 & 1.9723 & 129196 & 87 \\
260 & $4.4494 \mathrm{e}-49$ & 0.0041 & 1.9742 & 106298 & 86 \\
300 & $1.4765 \mathrm{e}-56$ & 0.0044 & 1.9767 & 88192 & 84 \\
340 & $4.8887 \mathrm{e}-64$ & 0.0047 & 1.9784 & 73833 & 82 \\
380 & $1.6223 \mathrm{e}-71$ & 0.0053 & 1.9792 & 62571 & 77 \\
420 & $5.5025 \mathrm{e}-79$ & 0.0059 & 1.9801 & 53472 & 73 \\
460 & $1.8175 \mathrm{e}-86$ & 0.0064 & 1.9811 & 45541 & 68 \\
500 & $5.9896 \mathrm{e}-94$ & 0.0066 & 1.9823 & 39072 & 64 \\
\hline
\end{tabular}

to $\mathbb{H}\left(\mathbf{x}, \mathrm{DW}^{\varepsilon, \delta}(\mathbf{x})\right) \approx \mathbb{H}\left(\mathbf{x}, \boldsymbol{\alpha}_{1}\right)$, which is negative for $x_{2}=0$ (and hence violates property 2 in Definition 2 ).

Acknowledgements This work is supported by the Netherlands Organization for Scientific Research (NWO), Project Number 613.001.105. 
Open Access This article is distributed under the terms of the Creative Commons Attribution 4.0 International License (http://creativecommons.org/licenses/by/4.0/), which permits unrestricted use, distribution, and reproduction in any medium, provided you give appropriate credit to the original author(s) and the source, provide a link to the Creative Commons license, and indicate if changes were made.

\section{References}

1. Buijsrogge, A., de Boer, P.T., Rosen, K., Scheinhardt, W.R.W.: Large deviations for the total queue size in non-Markovian tandem queues. Queueing Syst. 85(3), 305-312 (2017). https://doi.org/10.1007/ s11134-016-9512-z

2. Buijsrogge, A., de Boer, P.T., Scheinhardt, W.R.W.: On state-independent importance sampling for the $G I|G I| 1$ tandem queue. Probab. Eng. Inf. Sci. https://doi.org/10.1017/S0269964818000426

3. de Boer, P.T.: Analysis of state-independent importance-sampling measures for the two-node tandem queue. ACM Trans. Model. Comput. Simul. 16(3), 225-250 (2006)

4. de Boer, P.T., Scheinhardt, W.R.W.: Alternative proof and interpretations for a recent state-dependent importance sampling scheme. Queueing Syst. 57(2-3), 61-69 (2007)

5. Dean, T., Dupuis, P.: Splitting for rare event simulation: a large deviation approach to design and analysis. Stoch. Process. Their Appl. 119(2), 562-587 (2009)

6. Dupuis, P., Sezer, A.D., Wang, H.: Dynamic importance sampling for queueing networks. Ann. Appl. Probab. 17(4), 1306-1346 (2007)

7. Dupuis, P., Wang, H.: Subsolutions of an Isaacs equation and efficient schemes for importance sampling. Math. Oper. Res. 32(3), 723-757 (2007)

8. Dupuis, P., Wang, H.: Importance sampling for Jackson networks. Queueing Syst. 62(1), 113-157 (2009)

9. Frater, M.R., Anderson, B.D.O.: Fast simulation of buffer overflows in tandem networks of $G I|G I| 1$ queues. Ann. Oper. Res. 49, 207-220 (1994)

10. Glasserman, P., Kou, S.G.: Analysis of an importance sampling estimator for tandem queues. ACM Trans. Model. Comput. Simul. 5(1), 22-42 (1995)

11. Parekh, S., Walrand, J.: A quick simulation method for excessive backlogs in networks of queues. IEEE Trans. Autom. Control 34(1), 54-66 (1989)

12. Sadowsky, J.S.: Large deviations theory and efficient simulation of excessive backlogs in a $G I|G I| m$ queue. IEEE Trans. Autom. Control 36(12), 1383-1394 (1991)

Publisher's Note Springer Nature remains neutral with regard to jurisdictional claims in published maps and institutional affiliations. 